Louisiana State University

LSU Digital Commons

$11-1-2016$

\title{
The first circumbinary planet found by microlensing: OGLE-2007-BLG-349L(AB)c
}

\author{
D. P. Bennett \\ NASA Goddard Space Flight Center
}

S. H. Rhie

University of Notre Dame

A. Udalski

University of Warsaw

A. Gould

The Ohio State University

Y. Tsapras

Astronomisches Rechen-Institut Heidelberg

See next page for additional authors

Follow this and additional works at: https://digitalcommons.Isu.edu/physics_astronomy_pubs

\section{Recommended Citation}

Bennett, D., Rhie, S., Udalski, A., Gould, A., Tsapras, Y., Kubas, D., Bond, I., Greenhill, J., Cassan, A., Rattenbury, N., Boyajian, T., Luhn, J., Penny, M., Anderson, J., Abe, F., Bhattacharya, A., Botzler, C., Donachie, M., Freeman, M., Fukui, A., Hirao, Y., Itow, Y., Koshimoto, N., Li, M., Ling, C., Masuda, K., Matsubara, Y., Muraki, Y., Nagakane, M., Ohnishi, K., Oyokawa, H., Perrott, Y., \& Saito, T. (2016). The first circumbinary planet found by microlensing: OGLE-2007-BLG-349L(AB)c. Astronomical Journal, 152 (5) https://doi.org/10.3847/0004-6256/152/5/125

This Article is brought to you for free and open access by the Department of Physics \& Astronomy at LSU Digital Commons. It has been accepted for inclusion in Faculty Publications by an authorized administrator of LSU Digital Commons. For more information, please contact ir@lsu.edu. 


\section{Authors}

D. P. Bennett, S. H. Rhie, A. Udalski, A. Gould, Y. Tsapras, D. Kubas, I. A. Bond, J. Greenhill, A. Cassan, N. J. Rattenbury, T. S. Boyajian, J. Luhn, M. T. Penny, J. Anderson, F. Abe, A. Bhattacharya, C. S. Botzler, M. Donachie, M. Freeman, A. Fukui, Y. Hirao, Y. Itow, N. Koshimoto, M. C.A. Li, C. H. Ling, K. Masuda, Y. Matsubara, Y. Muraki, M. Nagakane, K. Ohnishi, H. Oyokawa, Y. C. Perrott, and To Saito 
THE FIRST CIRCUMBINARY PLANET FOUND BY MICROLENSING: OGLE-2007-BLG-349L(AB)c

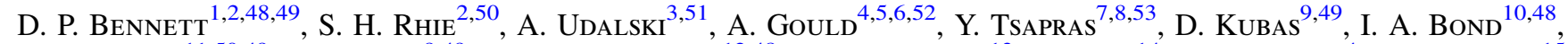

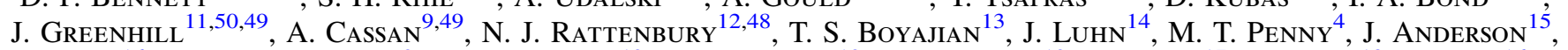
F. Abe $^{16}$, A. Bhattacharya ${ }^{2}$, C. S. Botzler ${ }^{12}$, M. Donachie ${ }^{12}$, M. Freeman $^{12}$, A. Fukui ${ }^{17}$, Y. Hirao ${ }^{18}$, Y. Itow $^{16}$, N. Koshimoto ${ }^{18}$, M. C. A. Li ${ }^{12}$, C. H. Ling ${ }^{9}$, K. Masuda ${ }^{16}$, Y. Matsubara ${ }^{16}$, Y. Muraki ${ }^{16}$, M. Nagakane ${ }^{18}$, K. Ohnishi ${ }^{19}$, H. Oyokawa ${ }^{16}$, Y. C. Perroti ${ }^{12}$, To. Saito ${ }^{20}$, A. Sharan ${ }^{12}$, D. J. Sullivan ${ }^{21}$, T. Sumi ${ }^{18}$, D. Suzuki ${ }^{1,2}$, P. J. Tristram ${ }^{22}$, A. YONEHARA ${ }^{23}$, P. C. M. YOCK ${ }^{12}$

(The MOA Collaboration),

M. K. SZYMAŃSKI ${ }^{3}$, I. SOSZYŃSKI ${ }^{3}$, K. UlACZYK $^{3}$, Ł. WYRZYKOWSKI $^{3}$

(The OGLE Collaboration),

W. Allen ${ }^{24}$, D. DePoy ${ }^{25}$, A. Gal-Yam ${ }^{26}$, B. S. Gaudi ${ }^{4}$, C. $\mathrm{HAN}^{27}$, I. A. G. Monard ${ }^{28}$, E. Ofek ${ }^{29}$, R. W. Pogge ${ }^{4}$

(The $\mu$ FUN COLlaboration),

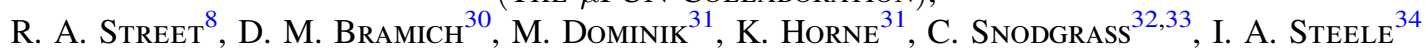

(The Robonet Collaboration),

AND

M. D. Albrow ${ }^{35}$, E. Bachelet $^{8}$, V. Batista ${ }^{9}$, J.-P. Beaulieu ${ }^{9}$, S. Brillant ${ }^{36}$, J. A. R. Caldwell ${ }^{37}$, A. Cole ${ }^{11}$, C. Coutures ${ }^{9}$, S. Dieters ${ }^{11}$, D. Dominis Prester ${ }^{38}$, J. Donatowicz ${ }^{39}$, P. Fouqué ${ }^{40,41}$, M. Hundertmark $^{31,42}$, U. G. Jørgensen ${ }^{42}$, N. Kains ${ }^{15}$, S. R. Kane ${ }^{43}$, J.-B. Marquette ${ }^{9}$, J. Menzies ${ }^{44}$, K. R. Pollard ${ }^{35}$, C. Ranc ${ }^{8}$, K. C. SAhu $^{15}$, J. Wambsganss ${ }^{45}$,

A. Williams ${ }^{46,47}$, M. ZuB ${ }^{45}$

(The Planet Collaboration)

${ }^{1}$ Code 667, NASA Goddard Space Flight Center, Greenbelt, MD 20771, USA; david.bennett@ nasa.gov

${ }^{2}$ Deptartment of Physics, University of Notre Dame, 225 Nieuwland Science Hall, Notre Dame, IN 46556, USA

${ }^{3}$ Warsaw University Observatory, Al. Ujazdowskie 4, 00-478 Warszawa, Poland

${ }^{4}$ Department of Astronomy, Ohio State University, 140 West 18th Avenue, Columbus, OH 43210, USA

${ }^{5}$ Max-Planck-Institute for Astronomy, Königstuhl 17, D-69117 Heidelberg, Germany

${ }^{6}$ Korea Astronomy and Space Science Institute, Daejon 34055, Republic Of Korea

${ }^{7}$ Astronomisches Rechen-Institut, Zentrum für Astronomie der Universität Heidelberg (ZAH), D-69120 Heidelberg, Germany

${ }^{8}$ Las Cumbres Observatory Global Telescope Network, 6740 Cortona Drive, Suite 102, Goleta, CA 93117, USA

${ }^{9}$ Institut d'Astrophysique de Paris, 98 bis bd Arago, 75014 Paris, France

${ }^{10}$ Institute of Natural and Mathematical Sciences, Massey University, Auckland 0745, New Zealand

${ }^{11}$ School of Math and Physics, University of Tasmania, Private Bag 37, GPO Hobart, 7001 Tasmania, Australia

12 Department of Physics, University of Auckland, Private Bag 92019, Auckland, New Zealand

${ }^{13}$ Department of Astronomy, Yale University, New Haven, CT 06511, USA

${ }^{14}$ Pennsylvania State University, 537 Davey Lab, University Park, PA 16802, USA

${ }^{15}$ Space Telescope Science Institute, 3700 San Martin Drive, Baltimore, MD 21218, USA

${ }^{16}$ Institute for Space-Earth Environmental Research, Nagoya University, Nagoya 464-8601, Japan

${ }^{17}$ Okayama Astrophysical Observatory, National Astronomical Observatory of Japan,

3037-5 Honjo, Kamogata, Asakuchi, Okayama 719-0232, Japan
${ }^{18}$ Department of Earth and Space Science, Graduate School of Science, Osaka University, Toyonaka, Osaka 560-0043, Japan

${ }_{19}$ Nagano National College of Technology, Nagano 381-8550, Japan

${ }^{20}$ Tokyo Metropolitan College of Aeronautics, Tokyo 116-8523, Japan

${ }^{21}$ School of Chemical and Physical Sciences, Victoria University, Wellington, New Zealand

${ }^{22}$ Mt. John University Observatory, P.O. Box 56, Lake Tekapo 8770, New Zealand

${ }^{23}$ Department of Physics, Faculty of Science, Kyoto Sangyo University, 603-8555 Kyoto, Japan

${ }^{25}$ Department of Physics, Texas A\&M University, 4242 TAMU, College Station, TX 77843-4242, USA

${ }^{26}$ Department of Particle Physics and Astrophysics, Weizmann Institute of Science, 234 Herzl Street, 76100 Rehovot, Israel

${ }^{27}$ Department of Physics, Chungbuk National University, Cheongju 361-763, Republic of Korea

${ }^{28}$ Bronberg and Kleinkaroo Observatories, Centre for Backyard Astrophysics, Calitzdorp, South Africa

${ }^{30}{ }^{29}$ Weizmann Institute of Science, 234 Herzl Street, Rehovot 7610001, Israel

${ }^{30}$ Qatar Environment and Energy Research Institute(QEERI), HBKU, Qatar Foundation, Doha, Qatar

${ }^{31}$ SUPA, School of Physics and Astronomy, University of St Andrews, North Haugh, St Andrews KY16 9SS, UK

${ }^{32}$ Planetary and Space Sciences, Department of Physical Sciences, The Open University, Milton Keynes, MK7 6AA, UK

${ }^{33}$ Max Planck Institute for Solar System Research, Justus-von-Liebig-Weg 3, D-37077 Göttingen, Germany

${ }^{34}$ Astrophysics Research Institute, Liverpool John Moores University, Liverpool CH41 1LD, UK

${ }^{35}$ University of Canterbury, Department of Physics and Astronomy, Private Bag 4800, 8020 Christchurch, New Zealand

${ }_{37}^{36}$ ESO Vitacura, Alonso de Córdova 3107, Vitacura, Casilla 19001, Santiago 19, Chile

${ }^{37}$ McDonald Observatory, 82 Mt. Locke Road, McDonald Observatory, TX 79734, USA

${ }^{38}$ Department of Physics, University of Rijeka, Radmile Matej vcić 2, 51000 Rijeka, Croatia

${ }^{39}$ Technical University of Vienna, Department of Computing, Wiedner Hauptstrasse 10, 1040 Wien, Austria

${ }^{40}$ CFHT Corporation, 65-1238 Mamalahoa Highway, Kamuela, HI 96743, USA

${ }^{41}$ IRAP, CNRS-Université de Toulouse, 14 av. E. Belin, F-31400 Toulouse, France

42 Niels Bohr Institutet, Københavns Universitet, Juliane Maries Vej 30, DK-2100 København Ø, Denmark

${ }^{43}$ Department of Physics and Astronomy, San Francisco State University, 1600 Holloway Avenue, San Francisco, CA 94132, USA

${ }^{44}$ South African Astronomical Observatory, P.O. Box 9, Observatory 7935, South Africa 


\author{
${ }^{45}$ Astronomisches Rechen-Institut, Zentrum für Astronomie der Universität Heidelberg (ZAH), \\ Mönchhofstrasse 12-14, D-69120 Heidelberg, Germany \\ ${ }^{46}$ Perth Observatory, Walnut Road, Bickley, Perth 6076, Australia \\ ${ }^{47}$ International Centre for Radio Astronomy Research, Curtin University, Bentley, WA 6102, Australia \\ Received 2016 April 25; revised 2016 August 23; accepted 2016 August 27; published 2016 October 21
}

\begin{abstract}
We present the analysis of the first circumbinary planet microlensing event, OGLE-2007-BLG-349. This event has a strong planetary signal that is best fit with a mass ratio of $q \approx 3.4 \times 10^{-4}$, but there is an additional signal due to an additional lens mass, either another planet or another star. We find acceptable light-curve fits with two classes of models: two-planet models (with a single host star) and circumbinary planet models. The light curve also reveals a significant microlensing parallax effect, which constrains the mass of the lens system to be $M_{L} \approx 0.7 M_{\odot}$. Hubble Space Telescope (HST) images resolve the lens and source stars from their neighbors and indicate excess flux due to the star(s) in the lens system. This is consistent with the predicted flux from the circumbinary models, where the lens mass is shared between two stars, but there is not enough flux to be consistent with the two-planet, one-star models. So, only the circumbinary models are consistent with the $H S T$ data. They indicate a planet of mass $m_{c}=80 \pm 13 M_{\oplus}$, orbiting a pair of $\mathrm{M}$ dwarfs with masses of $M_{A}=0.41 \pm 0.07$ and $M_{B}=0.30 \pm 0.07$, which makes this the lowest-mass circumbinary planet system known. The ratio of the separation between the planet and the center of mass to the separation of the two stars is $\sim 40$, so unlike most of the circumbinary planets found by Kepler, the planet does not orbit near the stability limit.
\end{abstract}

Key words: gravitational lensing: micro - planetary systems

\section{INTRODUCTION}

One of the main features of the observational study of extrasolar planets has been the continuing stream of surprise observational discoveries. These include planets orbiting a pulsar (Wolszczan \& Frail 1992); hot Jupiters (Mayor \& Queloz 1995); systems of short-period, low-density planets in tightly packed orbits (Lissauer 2011); and circumbinary planets (Doyle et al. 2011) close to the stability limit. Circumbinary planets and planets in close binary systems are very difficult to detect with the radial velocity method, but Kepler has proved quite adept at finding such systems (Doyle et al. 2011; Orosz et al. 2012; Welsh et al. 2012, 2015; Kostov et al. 2013, 2014, 2016). Gravitational microlensing (Bennett 2008; Gaudi 2012) has demonstrated the ability to detect such systems (Bennett et al. 1999; Gould et al. 2014; Poleski et al. 2014; Udalski et al. 2015) (either circumbinary planets or planets orbiting one member of a relatively close binary). Two of these claimed microlensing planets in binary systems have turned out to be incorrect, MACHO-97-BLG-41 (Bennett et al. 1999; Albrow et al. 2000; Jung et al. 2013) and OGLE-2013-BLG-0723 (Udalski et al. 2015; Han et al. 2016), but this is largely an issue that can be addressed by greater care in event modeling. These events still help to establish the sensitivity of the microlensing method to planets in close binary systems, because in each case the lightcurve measurements do definitively distinguish between the triple-lens models, planetary models, and the close binary models without a planet.

In this paper, we present the first circumbinary planet found by microlensing, OGLE-2007-BLG-349L(AB)c. ${ }^{54}$ The signal for this event is dominated by the microlensing effect of a

\footnotetext{
48 MOA Collaboration.

49 PLANET Collaboration.

50 Deceased.

51 OGLE Collaboration.

$52 \mu$ FUN Collaboration.

53 Robonet Collaboration.

54 Our designation for this event corrects an apparent inconsistency in the naming of planets in binary systems by using a unique letter for each mass in the system, following the convention for planets orbiting single stars.
}

Saturn-mass-ratio planet, but the very central part of the planetary binary-lens light curve does not fit the data. As we show in Section 3, the light curve can be fit by models with an additional lens mass, either another planet or another star. However, the light-curve data do not tell us which of these models is correct. Nevertheless, the light curve does reveal finite-source effects and a microlensing parallax signal that allow us to determine the lens system mass, as we discuss in Section 4.

In Section 4.2, we present Hubble Space Telescope (HST) observations of the OGLE-2007-BLG-349 lens system and source star. These observations clearly indicate excess flux at the position of the source, which is consistent with the circumbinary models but not the two-planet models. If the stellar mass of the lens system is divided into two masses, then it is substantially fainter ( 1.6 mag) in the $I$ band than a single host star would be. And it is only such a faint lens system that is consistent with the HST images, and so it is the HST observations that select the circumbinary model over the twoplanet models. In Section 4.3, we add the lens brightness constraint to our light-curve modeling in order to confirm this conclusion, and we find that two-planet models with an extremely faint host star (presumably a white dwarf) do better than the best two-planet models with a main-sequence host star. However, these models are still substantially worse than the circumbinary models, so they are excluded.

We consider adaptive optics observations of the source and lens stars in Section 5, and we find that these observations provide marginal support for the circumbinary interpretation of this light curve. Finally, in Section 6, we discuss the implications of this discovery for our understanding of the properties of exoplanets.

\section{LIGHT-CURVE DATA AND PHOTOMETRY}

Microlensing event OGLE-2007-BLG-349, at R. A. $=18: 05: 24.43$, decl. $=-26: 25: 19.0$, and Galactic coordinates $(l, b)=(4.3802,-2.5161)$, was identified as a microlensing candidate by the Optical Gravitational Lensing Experiment (OGLE) Collaboration Early Warning System 
Table 1

Error-bar Modification Parameters

\begin{tabular}{lcc}
\hline \hline Data Set & $K$ & $\sigma_{\text {min }}$ \\
\hline OGLE- $I$ & 0.979 & 0.006 \\
MOA- $(R+I)$ & 0.932 & 0.007 \\
CTIO- $I$ & 1.500 & 0.003 \\
CTIO- $H$ & 2.142 & 0.003 \\
FTN- $R$ & 2.598 & 0.003 \\
Palomar- $I$ & 1.605 & 0.004 \\
Canopus- $I$ & 0.974 & 0.002 \\
Bronberg- $U n$ & 1.048 & 0.012 \\
VLO- $U n$ & 1.312 & 0.008 \\
\hline
\end{tabular}

Note. Passband Un refers to unfiltered imaging.

(Udalski et al. 1994) and announced on 2007 July 2. Later that month, the event was independently identified and announced by the MOA Collaboration as MOA-2007-BLG-379. In mid- to late August, this event was recognized as a potential highmagnification event, with high sensitivity to planets, by the $\mu$ FUN, Robonet, and PLANET microlensing follow-up groups, so they started observations prior to peak magnification. On 2007 September 4, the planetary anomaly was first identified in the OGLE data by the $\mu$ FUN and OGLE groups at $\mathrm{HJD}^{\prime}=\mathrm{HJD}-2450000=4348.5$. Despite the fact that this event occurred near the end of the Galactic bulge observing season, we achieved nearly complete coverage of the lightcurve peak, with the largest data gap of only 55 minutes over a period of $22 \mathrm{hr}$ centered on the light-curve peaks. This coverage was achieved with the combined data of the OGLE and MOA survey groups and the $\mu \mathrm{FUN}$, Robonet, and PLANET microlensing follow-up groups.

The data set we use in this analysis consists of microlensing survey data from the OGLE $1.3 \mathrm{~m}$ telescope in Chile in the $I$ band and the MOA $1.8 \mathrm{~m}$ telescope in New Zealand in the custom MOA $R$ band, which is equivalent to the sum of the Cousins $R+I$ bands, as well as data from six telescopes operated by microlensing follow-up groups. Four of these telescopes are operated by the Microlensing Follow-up Network $(\mu \mathrm{FUN}) . \mu \mathrm{FUN}$ provided $V$-, I-, and $H$-band data from the $1.3 \mathrm{~m}$ SMARTS telescope at CTIO in Chile, I-band data from the $1.5 \mathrm{~m}$ Palomar telescope in California, and unfiltered data from the $0.35 \mathrm{~m}$ Bronberg Observatory telescope in South Africa and the $0.4 \mathrm{~m}$ Vintage Lane Observatory (VLO) telescope in New Zealand. The RoboNet Collaboration has provided $R$-band data from the $2 \mathrm{~m}$ Faulkes North Telescope (FTN), and the Probing Lensing Anomalies NETwork (PLANET) Collaboration provided I-band data from the $1.0 \mathrm{~m}$ Canopus Observatory telescope. We exclude from the analysis data from several $\mu \mathrm{FUN}$ observatories that were unable to obtain data near the light-curve peak.

The data were reduced with various implementations of the difference imaging method (Tomaney \& Crotts 1996). The MOA and OGLE data were reduced with their respective pipelines (Bond et al. 2001; Udalski 2003). The PLANET data were reduced with a version of ISIS (Alard \& Lupton 1998), and the RoboNet data were reduced with the RoboNet pipeline (Bramich 2008). Most of the $\mu \mathrm{FUN}$ data were reduced with the OGLE pipeline, but the CTIO $H$-band data were reduced with PySIS (Albrow et al. 2009).

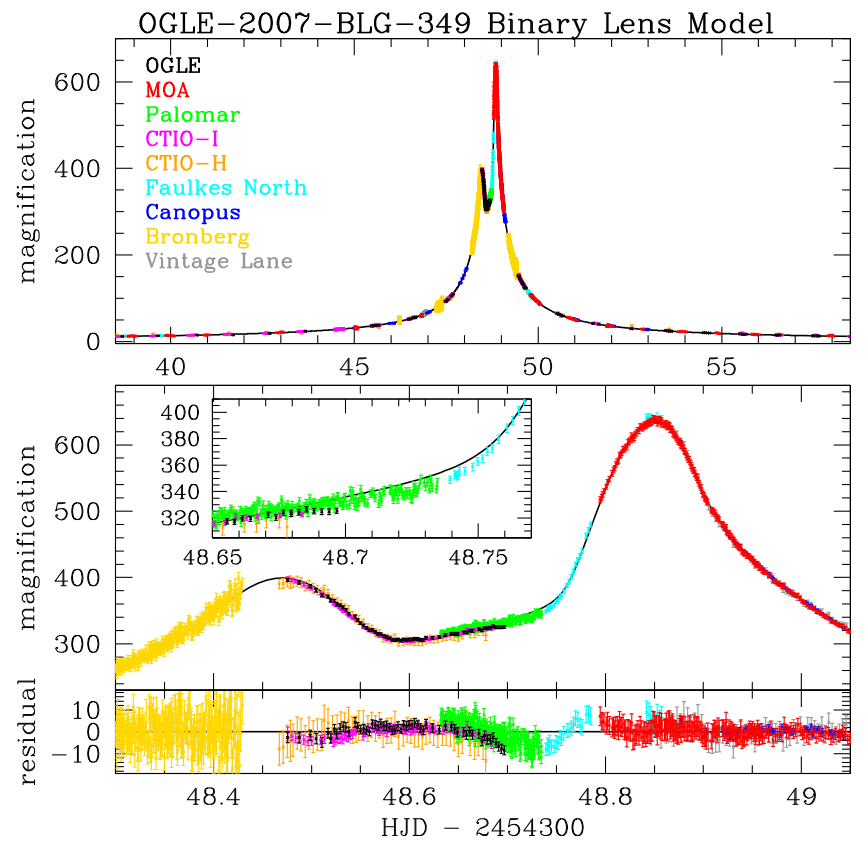

Figure 1. Best-fit one-star plus one-planet light curve. The top panel shows the 20 days centered on the light-curve peak, and the middle and bottom panels show the light curves and the residuals for the central day of the light curve. The single-planet model matches all the major light-curve features, but there are significant residuals at $48.65 \lesssim \mathrm{HJD}-2,454,300 \lesssim 48.82$. This is quite close to the $t_{0}$ value of the fit, which is the time when a light-curve feature due to an additional mass would be expected.

We follow the usual method (Yee et al. 2012) to improve the photometric error bars with the following formula:

$$
\sigma=K \sqrt{\sigma_{0}^{2}+\sigma_{\min }^{2}}
$$

where $\sigma_{0}$ is the error-bar estimate provided by the photometry code. The error bars in Equation (1) are in linear units as a fraction of the measured flux. For photometry provided in magnitudes, the error bars are converted to linear units prior to the Equation (1) modifications. The error-bar correction parameters $K$ and $\sigma_{\min }$ for each data set are listed in Table 1. These error-bar modifications are made based on an approximately correct reference model to give $\chi^{2} /$ dof $=1$ for each data set. The corrected error bars are normally then used to make more accurate estimates for the uncertainties in the physical parameters of the lens system, and the selection of the correct model does not depend on the error-bar corrections. In this case, however, there are competing models, so one might be concerned that the final conclusions could be dependent on which model light curve is used to determine the error-bar modification parameters. Fortunately, in this case, the competing two-planet and circumbinary light curves are so similar that the choice of the reference model does not have a significant effect on our analysis. The error-bar modifications are essentially independent of the reference model.

\section{LIGHT-CURVE MODELS}

The preliminary modeling of this event was done independently using the methods of Dong et al. (2006, 2009b) and Bennett (2010) to first search for the parameters of the planet that dominates the anomaly signal. This light curve is strongly 
Table 2

Best-fit Model Parameters

\begin{tabular}{|c|c|c|c|c|}
\hline Parameter & Units & One-planet & Two-planet & Circumbinary \\
\hline$t_{E}$ & days & 116.703 & 113.520 & 117.720 \\
\hline$t_{0}$ & $\mathrm{HJD}^{\prime}$ & 4348.7534 & 4348.7469 & 4348.7465 \\
\hline$u_{0}$ & $\ldots$ & -0.0021516 & 0.0020581 & -0.0019818 \\
\hline$d_{23}$ & $\ldots$ & $\ldots$ & 0.95046 & 0.019905 \\
\hline$\theta_{1 \mathrm{~cm}}$ & radians & 4.40140 & 1.89437 & 4.35940 \\
\hline$\phi_{23}$ & radians & $\ldots$ & -3.07611 & 0.36989 \\
\hline$\epsilon_{3}$ & $\cdots$ & $\cdots$ & 0.999615 & 0.53487 \\
\hline$t_{*}$ & days & 0.06614 & 0.06930 & 0.07064 \\
\hline$\dot{d}_{23 x}$ & days $^{-1}$ & $\cdots$ & 0.0 & 0.010478 \\
\hline$\dot{d}_{23 y}$ & days $^{-1}$ & $\cdots$ & 0.0 & -0.006360 \\
\hline $1 / T_{\text {orb }}$ & days $^{-1}$ & $\ldots$ & 0.0 & 0.059380 \\
\hline$\pi_{E}$ & $\ldots$ & 0.09693 & 0.19070 & 0.17458 \\
\hline$I_{L}$ & mag & $\cdots$ & 19.634 & 21.465 \\
\hline$I_{\mathrm{SL}}\left(t_{H 2}\right)$ & mag & $\ldots$ & 19.162 & 20.009 \\
\hline$V_{S}$ & mag & 22.367 & 22.334 & 22.375 \\
\hline$H_{S}$ & mag & 18.260 & 18.226 & 18.267 \\
\hline fit $\chi^{2}$ & $\ldots$ & 4237.56 & 3382.64 & 3382.25 \\
\hline dof & $\ldots$ & 3571 & 3568 & 3566 \\
\hline
\end{tabular}

Note. $\mathrm{HJD}^{\prime}=\mathrm{HJD}-2,450,000$. The reference time for the microlensing parallax and orbital motion parameters is $t_{\mathrm{fix}}=4349$.

dominated by the signal of a Saturn-mass-ratio planet with parameters quite similar to the model circulated by one of us (D.P.B.) within $24 \mathrm{hr}$ of the first detection of the planetary anomaly. (The basic geometry of the event was identified even earlier by two of us: A.C. and N.J.R.)

The best-fit binary-lens (one star + one planet) model is shown in Figure 1, and the parameters of this model are given in Table 2, including a significant microlensing parallax signal. (We use polar coordinates for the microlensing parallax vector, such that $\pi_{E, N}=\pi_{E} \cos \phi_{E}$ and $\pi_{E, E}=\pi_{E} \sin \phi_{E}$.) This model provides a good fit to most of the light-curve peak, but it does not fit the central part of the light curve at $48.65 \lesssim$ HJD $-2,454,300 \lesssim 48.82$, or $t \approx t_{0}$. This is the part of the light curve where we would expect to see the signal of another lens mass: a second planet or a stellar binary companion to the host star. So, we performed another initial condition grid search to explore possible triple-lens models. The triple-lens modeling was made possible by the theoretical work of Rhie (1997, 2002), which was particularly important for the modeling of orbital motion in a triple-lens system (Bennett et al. 2010). We fixed the parameters describing the best-fit planetary binary model and did the grid search over the parameters that describe the additional mass. There are three additional parameters for triple-lens models: two parameters describing the position of the third mass and one parameter describing its mass fraction. Using both methods, we search for three categories of solutions: two-planet models (with a single host star), models with the planet orbiting one member of a wide stellar binary, and models with a close stellar binary orbited by a circumbinary planet. Our initial triple-lens fits were done with static models, but the period of the stellar binaries for the circumbinary planet models will only be $\sim 10$ days. Since the duration of the light-curve peak is $\sim 0.5$ days, binary orbital motion is likely to be important for these circumbinary models. So, after finding the best-fit static circumbinary models, we include orbital motion of the two stars for these models. The circumbinary models include three additional parameters: the two-dimensional velocity in the plane of the sky and the inverse of the orbital period. However, these orbital parameters are also subject to a constraint, described below in Section 4.

All three categories of models that we explore can provide a substantial improvement to the light curve over the singleplanet model, but only the two-planet and circumbinary models (with orbital motion) can provide a good fit to the light-curve data. The best-fit two-planet and circumbinary model parameters are given in Table 2, and the best-fit light curves are shown in Figure 2. The parameters we use are the same as used in the analysis of the first triple-lens microlensing event, the two-planet event, OGLE-2006-BLG-109 (Gaudi et al. 2008; Bennett et al. 2010). The coordinates are based on the centerof-mass system, with a system of total mass $M$. The length parameters are normalized by the Einstein radius of this total system mass, $R_{E}=\sqrt{\left(4 G M / c^{2}\right) D_{S} x(1-x)}$, where $x=D_{L} /$ $D_{S}$ and $D_{L}$ and $D_{S}$ are the lens and source distances, respectively. ( $G$ and $c$ are the gravitational constant and speed of light, as usual.) $t_{E}$ is the Einstein radius crossing time, while $t_{0}$ and $u_{0}$ are the time and separation of closest approach of the source to the center of mass. The separation between mass 1 and the center of mass of masses 2 and 3 is given by $d_{1 \mathrm{~cm}}$, and $d_{23}$ is the distance between masses 2 and 3 . The lens axis is defined as the vector between mass 1 and the mass $2+3$ center of mass, and $\theta_{1 \mathrm{~cm}}$ is the angle between the source trajectory and 

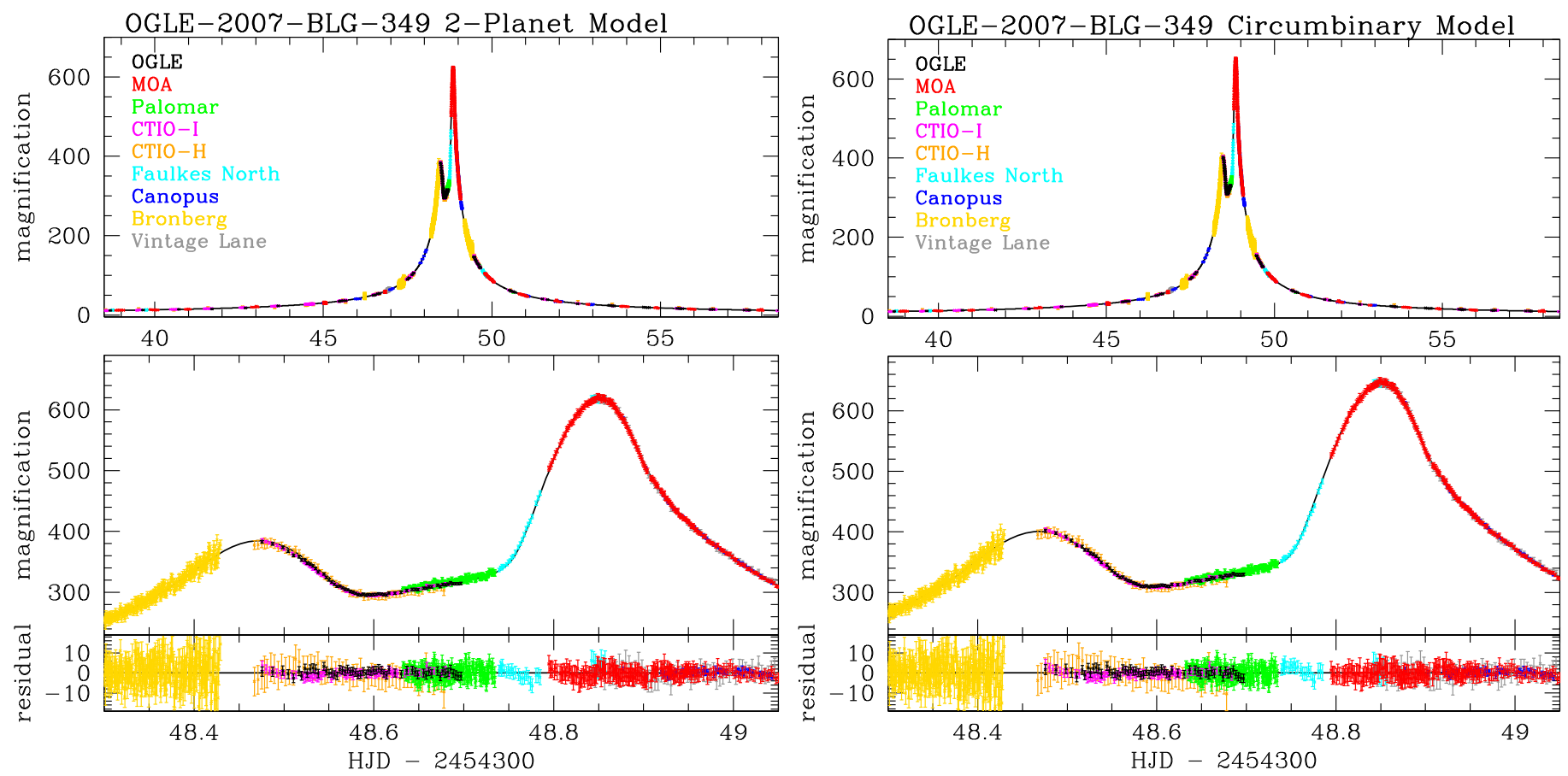

Figure 2. Best-fit two-planet (left) and circumbinary (right) light curves with the parameters given in Table 2. Both models fit the light curve almost equally well.
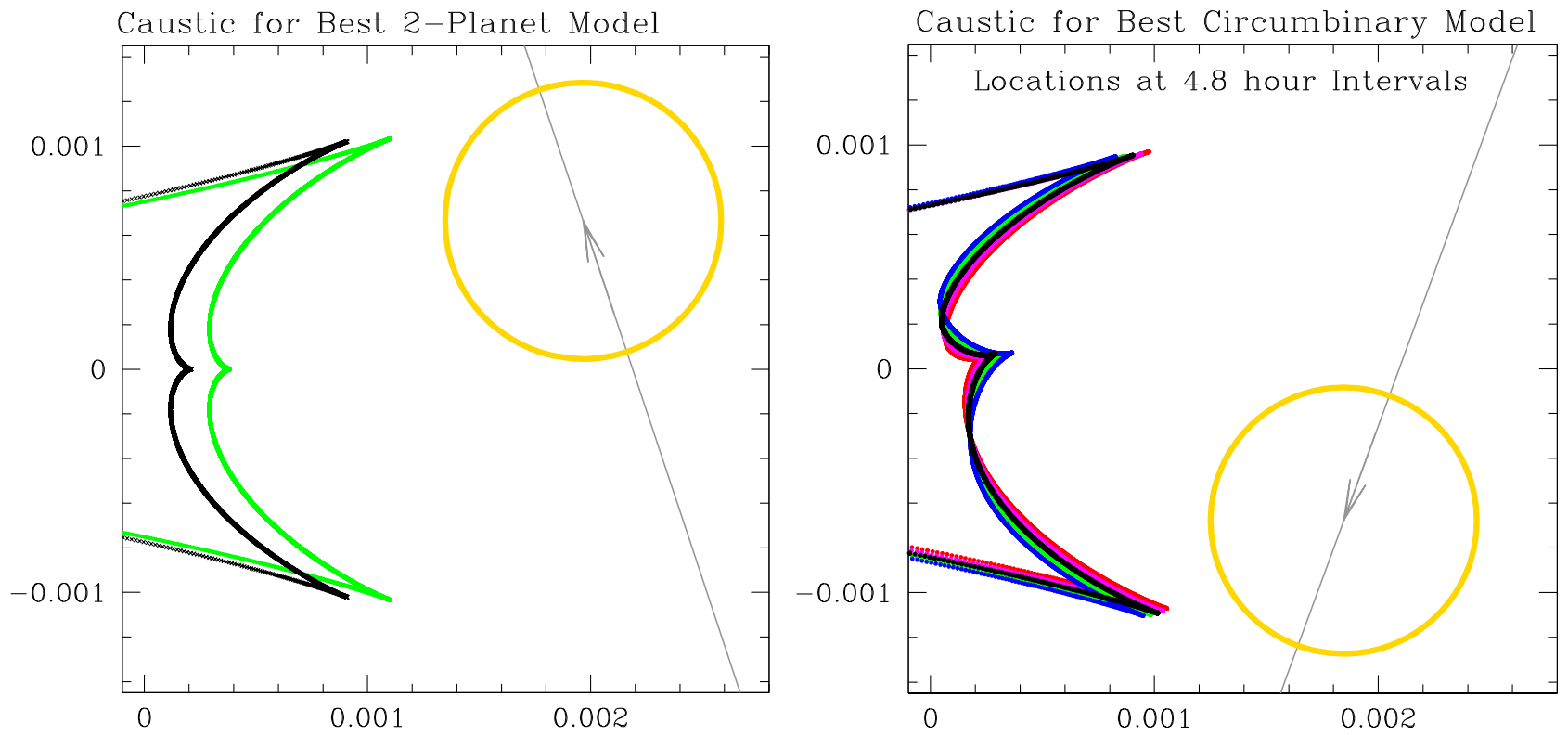

Figure 3. OGLE-2007-BLG-349 caustics for the best one-planet model (in green) and the best two-planet model (in black) on the left and for the best-fit circumbinary planet model at $4.8 \mathrm{hr}$ intervals on the right. The gold circle indicates the source size, and the gray line indicates the source trajectory, with an arrow indicating the source position at $t=t_{0}$ and direction of motion.

the lens axis, while $\phi_{23}$ is the angle between the line connecting masses 2 and 3 and the lens axis. The mass fractions of each of the three masses are $\epsilon_{1}, \epsilon_{2}$, and $\epsilon_{3}$, but these parameters are not independent since $\epsilon_{1}+\epsilon_{2}+\epsilon_{3} \equiv 1$. The source radius crossing time is given by $t_{*}$. Microlensing parallax is described by $\pi_{E}$ and $\phi_{E}$, as described above. The orbital motion of masses 2 and 3 is described by three parameters. The instantaneous velocity along the lens axes is given by $\dot{d}_{23 x}$, while $\dot{d}_{23 y}$ gives the velocity perpendicular to the lens axis. The orbits are constrained to be circular with a period of $T_{\text {orb }}$, and we use $1 / T_{\text {orb }}$ as a fit parameter. (In our models, mass 1 refers to the primary planet, mass 3 refers to a host star, and mass 2 can be either a second host star or a second planet.)

As can be seen from Table 2 and Figure 2, the light-curve data do not distinguish between the best two-planet and circumbinary models. The $\chi^{2}$ values for the two models are nearly identical, with the best circumbinary model favored over the best two-planet model by $\Delta \chi^{2}=0.39$, but the two-planet model has two more degrees of freedom, because of the three additional orbital parameters and one constraint to be explained below in Section 4. Either model would represent a remarkable discovery. This could be the first circumbinary planet found by 

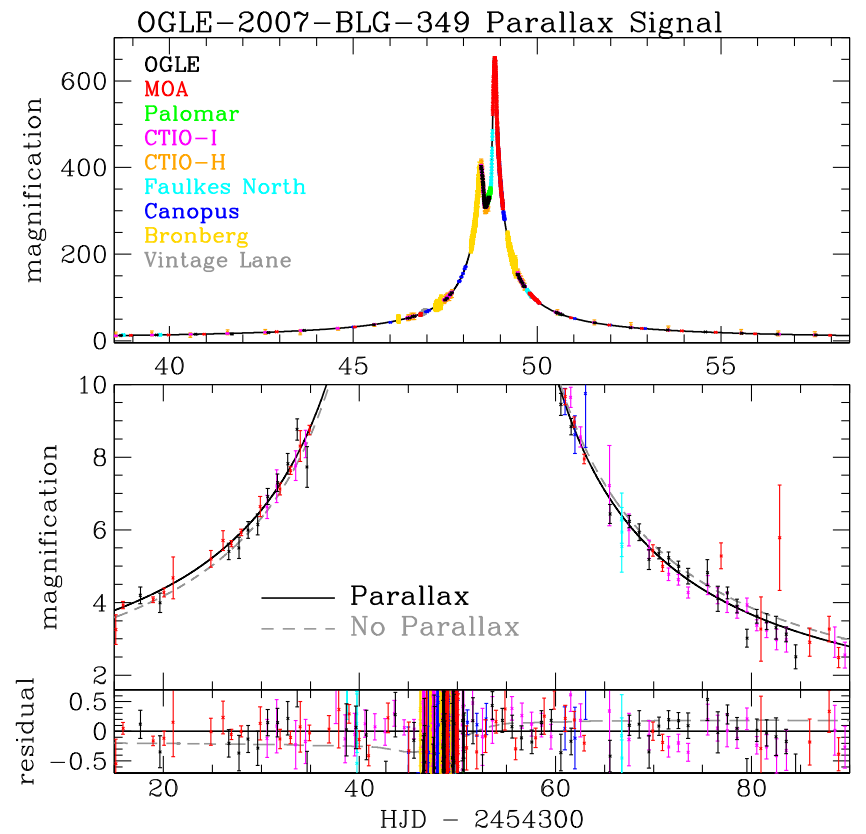

Figure 4. Comparison of the best two-planet microlensing model with and without microlensing parallax plotted as solid black and dashed gray curves, respectively. Much of the parallax signal comes in the moderate magnification wings of the light curve. From the bottom panel, we can see that the data are well above the no-parallax light curve prior to the peak and below the noparallax light curve after the peak.

microlensing or the first microlens planet with a mass ratio of $<10-6$ demonstrating microlensing's sensitivity to Earth-mass planets (Bennett \& Rhie 1996).

Both the two-planet and circumbinary models appear to fit the light-curve data equally well, but there are subtle differences that are apparent in the residuals plotted in the bottom panel of each light-curve figure. These residual panels also reveal low-level systematic discrepancies between the different data sets.

Figure 3 shows close-ups of the caustic configuration for the three best-fit models with the source trajectories given by the gray lines. The orbital motion of the two stars causes the caustics to move for the circumbinary model. They are displayed at $4.8 \mathrm{hr}$ intervals starting at $t=4348.25$ in units of $\mathrm{HJD}^{\prime}=\mathrm{HJD}-2,450,000$. The sequence of caustic curves is red, magenta, black, cyan, and blue.

\subsection{Microlensing Parallax}

An important feature of the OGLE-2007-BLG-349 light curve is the microlensing parallax signal. We find that the microlensing parallax effect improves the $\chi^{2}$ by $\Delta \chi^{2}=152.8$ as indicated in Figure 4. The parallax signal is quite clear in the second and third panels of this figure. Figure 5 shows the cumulative difference between the best-fit parallax and nonparallax models. This indicates that the parallax signal is centered between the time of the peak and the time of the maximum acceleration of Earth (by the Sun) in the directions perpendicular to the line of sight, as expected for a real microlensing parallax signal.

There are two contributions to the microlensing parallax signal: orbital parallax due to the Earth's orbital motion around the Sun (Gould 1992; Alcock et al. 1995) and terrestrial parallax (Gould et al. 2009), due to observations from

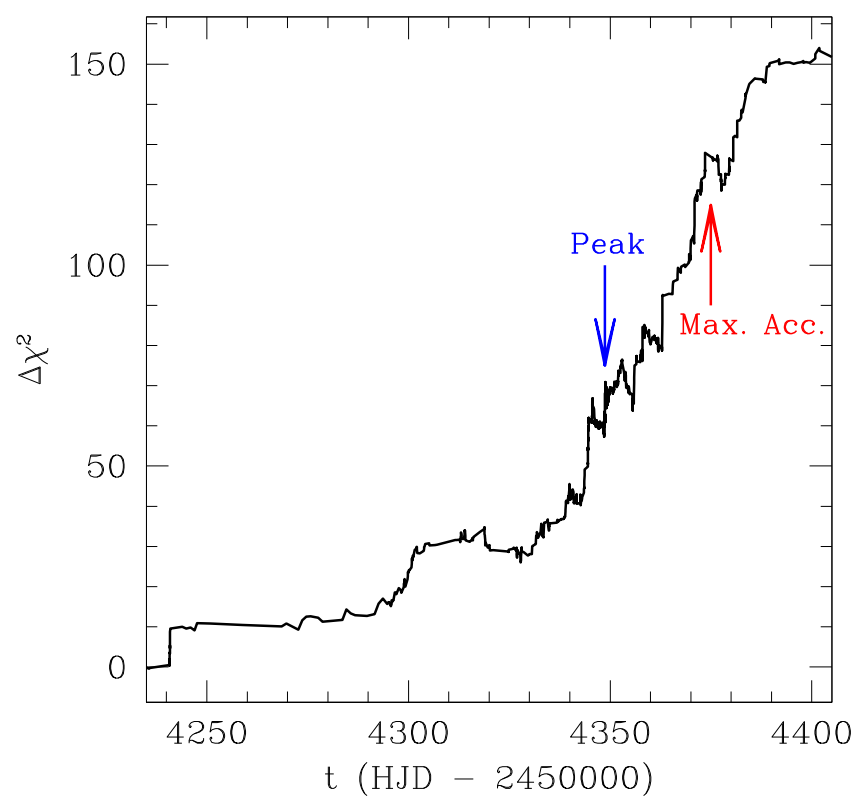

Figure 5. Difference in the cumulative $\Delta \chi^{2}$ between the best-fit nonparallax and parallax circumbinary models as a function of time, with the final $\Delta \chi^{2}=152.8$ for the full light curve. This indicates that the signal is centered between the light-curve peak and the time of maximum acceleration of Earth in the direction perpendicular to the line of sight. This is exactly where we expect the signal to be strongest.

telescopes at different locations on the Earth. The measurement of orbital parallax is fairly common, particularly for events like OGLE-2007-BLG-349 with durations $t_{E}>100$ days that occur near the beginning or end of the Galactic bulge observing season, when the acceleration of Earth was nearly perpendicular to the line of sight to the bulge. (OGLE-2007-BLG-349 reached peak magnification on 2007 September 5, just about 3 weeks before the acceleration of Earth is perpendicular to the line of sight). The orbital parallax signal is much stronger than the terrestrial parallax signal and is dominated by the three data sets that observed the event at modest magnification, MOA, OGLE, and $\mu$ FUN-CTIO, with $\Delta \chi^{2}$ values of $46.6,46.0$, and 54.1, respectively. Since the acceleration of Earth is almost entirely in the east-west direction, the east component of the orbital parallax solution is much more strongly constrained than the north component.

Terrestrial parallax is normally quite difficult to measure because the Einstein radius projected to the position of the solar system, $\tilde{r}_{E}$, is usually a few au or more, which is a few $\times 10^{5}$ larger than the separation of telescopes on the ground. For ultra-high-magnification events with a relatively large $\pi_{E}$ value, like OGLE-2007-BLG-224 (Gould et al. 2009), with a peak magnification of $A_{\max }>2000$, the signal can become quite strong. For events like OGLE-2007-BLG-349, presented in this paper, the terrestrial parallax signal is detectable, but relatively weak. However, terrestrial parallax does not have the strong east-west bias that orbital parallax has (Muraki et al. 2011). With data at or near the light-curve peak from northern hemisphere telescopes, like the FTS in Hawaii, along with southern hemisphere telescopes, like the MOA telescope in New Zealand and the CTIO and OGLE telescopes in Chile, we have some leverage on the north-south component of terrestrial parallax. So, the terrestrial parallax helps to constrain the north component of $\pi_{E}$, which is weakly constrained by orbital parallax. 

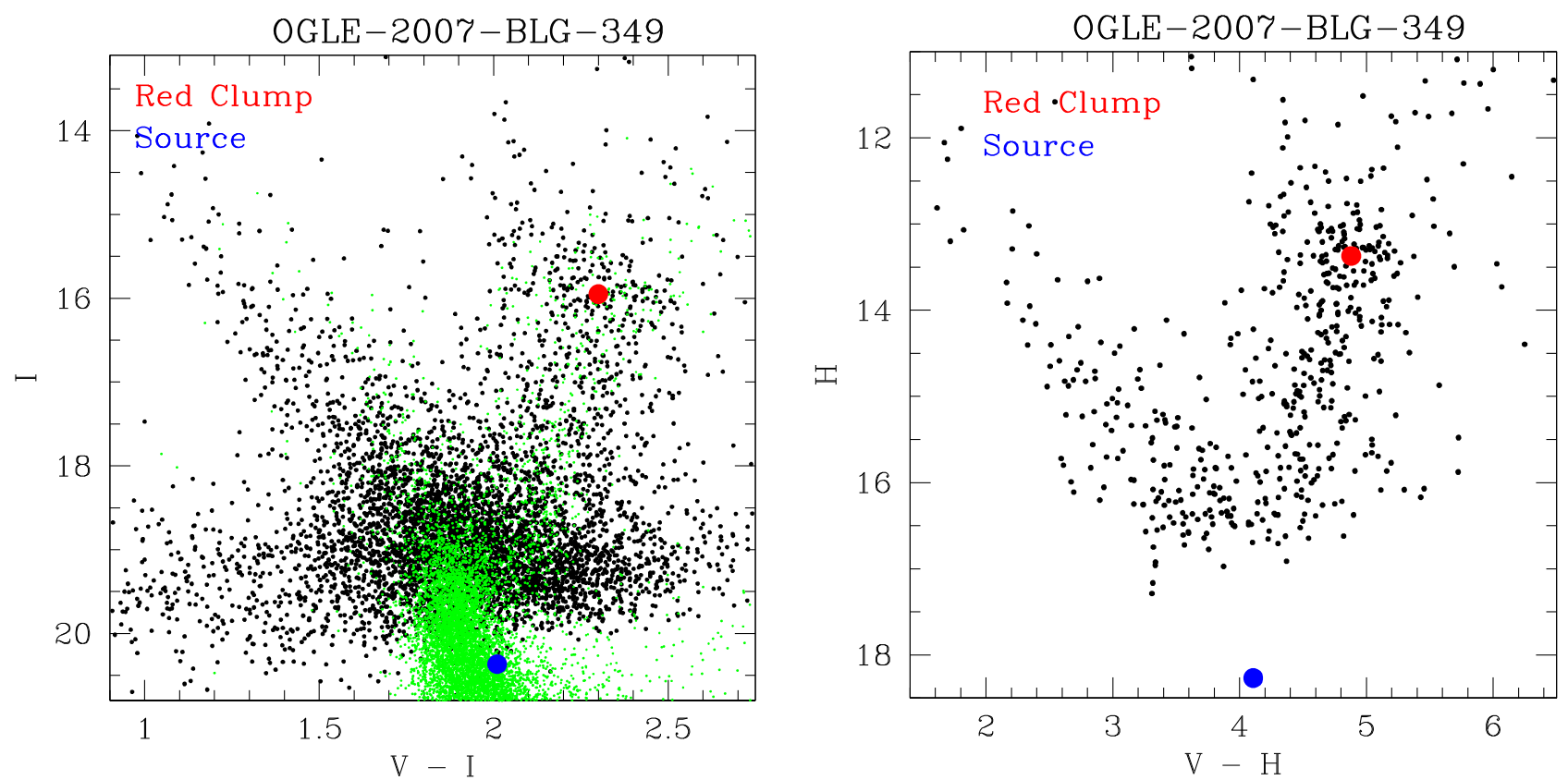

Figure 6. $(V-I, I)$ and $(V-H), H$ CMDs of the stars in the OGLE-III catalog (Szymański et al. 2011) within 90" of OGLE-2007-BLG-349. The green points are the Baade's Window CMD from Holtzman et al. (1998) shifted to the same extinction and distance as the OGLE-2007-BLG-349 field. The $H$-band magnitudes shown in the right panel come from IRSF images that have been calibrated to 2MASS. The red spots indicate the red clump giant centroid, and the blue spots indicate the source magnitudes and colors.

High-magnification events usually have several degeneracies. There is a degeneracy between close and wide solutions with $d_{1 \mathrm{~cm}} \simeq 0.81$ and $d_{1 \mathrm{~cm}} \simeq 1.23$, respectively. There is also a degeneracy between $u_{0}>0$ and $u_{0}<0$ solutions that would be exact if there was no microlensing parallax (representing the two reflections of the lens plane with respect to the projected orbit of Earth). In this case, the $u_{0}>0$ are excluded by the terrestrial parallax signal. The best $u_{0}>0$ and $u_{0}<0$ solutions have nearly identical $\chi^{2}$ values when terrestrial parallax is excluded from the modeling, but the $u_{0}>0$ models are disfavored by $\Delta \chi^{2}=28$ when we include terrestrial parallax. This difference in $\chi^{2}$ comes from the FTS, CTIO, MOA, and OGLE telescopes. We will explore these alternative models in more detail after applying the $H S T$ constraints on the lens system brightness.

\section{LENS SYSTEM PROPERTIES}

For events with measurable microlensing parallax signals, it is possible to determine the lens system mass if the angular Einstein radius, $\theta_{E}$, can also be determined (Gould 1992; An et al. 2002),

$$
M_{L}=\frac{\theta_{E} c^{2} \mathrm{AU}}{4 G \pi_{E}}=\frac{\theta_{E}}{(8.1439 \text { mas }) \pi_{E}} M_{\odot}
$$

Thus, we require the determination of the angular Einstein radius in order to determine the lens system mass. Fortunately, the sharp planetary light-curve features enable a precise measurement of the source radius crossing time, $t_{*}$. This provides a determination of the angular Einstein radius, $\theta_{E}=\theta_{*} t_{E} / t_{*}$, if we know the angular radius of the source star, $\theta_{*}$, which can be determined from the dereddened source magnitude and color (Kervella et al. 2004; Boyajian et al. 2014; Adams et al. 2016). We determine $\theta_{*}$ in Section 4.1. The lens system distance can also be determined from $\pi_{E}$ and $\theta_{E}$,

$$
D_{L}=\frac{1}{\pi_{\mathrm{E}} \theta_{\mathrm{E}}+\pi_{S}},
$$

assuming that the distance to the source, $D_{S}=1 / \pi_{S}$ (and its parallax, $\pi_{S}$ ), is known.

\subsection{Calibration and Source Radius}

Figure 6 shows color-magnitude diagrams (CMDs) of stars within $90^{\prime \prime}$ of the OGLE-2007-BLG-349 microlensing event. The green points in the left panel are from the Holtzman et al. (1998) Baade's Window CMD shifted to the same extinction and average distance as the OGLE-2007-BLG-349 field. The $V$ and $I$ magnitudes (indicated in black in the left panel) come from the OGLE-III photometry catalog (Szymański et al. 2011), and the $H$-band magnitudes come from images from the IRSF telescope that have been calibrated to the Two Micron All Sky Survey (2MASS) catalog (Carpenter 2001). The stars identified in these IRSF images have been crossmatched to the OGLE-III catalog, but not every star gives a good match. The IRSF images were taken in worse seeing than the OGLE-III catalog images, so some of the matches between the $V I$ - and $H$-band photometry have uncertainties due to blending where stars resolved in the OGLE images appear likely to be blended in the IRSF photometry. We do not include these stars in our $(V-H), H$ CMD, so the number of stars included in this CMD is smaller than in the $(V-I, I)$ CMD. (The OGLE-III $V-I$ CMD includes 9421 stars, but only 317 of the brighter stars have matched one to one with the stars seen in the $H$ band.)

These CMDs allow us to estimate the extinction toward the field centered on the source star location. From these CMDs (and the $(I-H), H$ CMD, which is not shown), we identify the centroid of the red clump giant distribution at $I_{\mathrm{rc}}=15.95 \pm 0.10, \quad\left(V_{\mathrm{rc}}-I_{\mathrm{rc}}\right)=2.30 \pm 0.05, \quad\left(V_{\mathrm{rc}}-H_{\mathrm{rc}}\right)$ 


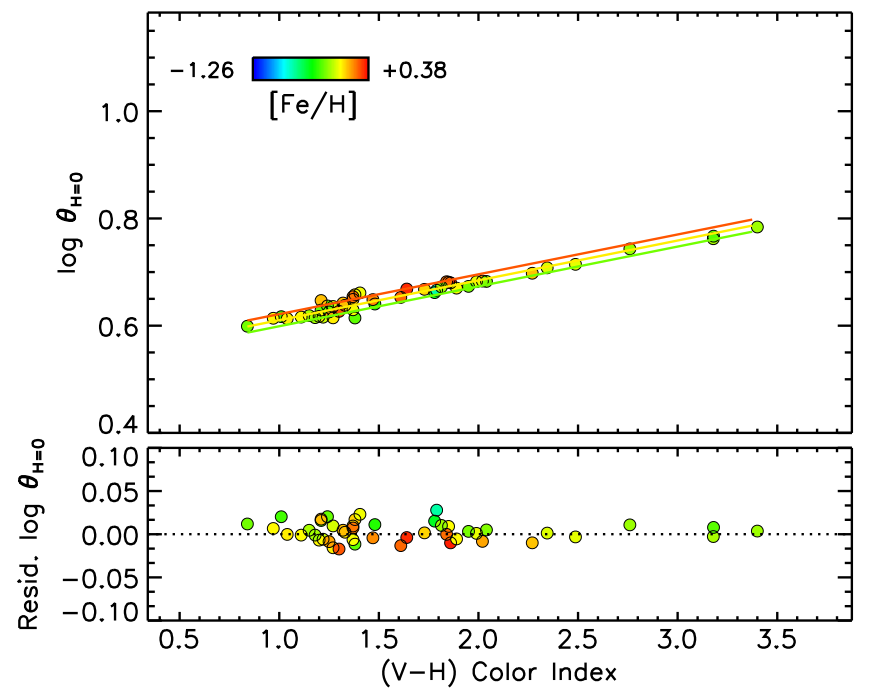

Figure 7. The $V-H, H$ angular source size relation from the analysis of Boyajian et al. (2014), including the effect of metallicity.

$=4.88 \pm 0.15$, and $\left(I_{\mathrm{rc}}-H_{\mathrm{rc}}\right)=2.58 \pm 0.10$. These are compared to the assumed intrinsic (dereddened) properties of red clump giant stars (Bennett et al. 2010; Nataf et al. 2013), $\quad M_{\mathrm{Irc}}=-0.13 \pm 0.10, \quad(V-I)_{\mathrm{rc} 0}=1.06 \pm$ $0.05, \quad(V-H)_{\mathrm{rc} 0}=2.23 \pm 0.07$, and $(I-H)_{\mathrm{rc} 0}=1.17 \pm$ 0.07. Fitting these constraints to the Cardelli et al. (1989) extinction law gives $R_{\mathrm{v}}=3.033, A_{H}=0.541, A_{I}=1.818$, and $A_{V}=3.083$.

The $V$ and $I$ source magnitudes were determined by calibrating the CTIO $V$ and $I$ light curves to the OGLE-III catalog (Szymański et al. 2011), and $H$ source magnitudes were determined by calibrating to the 2MASS-calibrated IRSF photometry. The $V$ and $I$ calibrations were done using DoPHOT (Schechter et al. 1993) light curves in order to put them on the same photometric scale as the CTIO CMD that was matched to the OGLE-III CMD shown in Figure 6, while the CTIO $H$-band calibrations were done with a SoDoPHOT reduction (Bennett et al. 1993) for the same reason. (Note that the OGLE-III light-curve photometry is not on the same scale as the OGLE-III catalog, and an OGLE CMD on the same scale as the OGLE-III light curve data was not available.) The calibrated source magnitudes, $V_{S}, I_{S}$, and $H_{S}$, for the best unconstrained models are displayed in Table 2.

With calibrated source magnitudes and an estimate of the extinction, we are now nearly ready to determine the angular source radius, using a color-angular size relation such as that of Kervella et al. (2004) or Boyajian et al. (2014), but in fact, we have more information about the source star. Cohen et al. (2008) took advantage of the extremely high magnification of this event to obtain a high-resolution spectrum of the source star, when it was magnified by a factor of $\sim 400$. This allows the metallicity of the source star to be determined, and we use the determination by Bensby et al. (2013), who find $[\mathrm{Fe} / \mathrm{H}]=+0.42 \pm 0.26$. This high metallicity is consistent with the CMD location of the source on the red edge of the bulge main sequence (as represented by source position with respect to the Baade's Window stars in Figure 6). Since metallicity is known to perturb the color-angular size relations, we asked the authors of Boyajian et al. (2014) to derive a relation using the dereddened $H$ and $V$ magnitudes including the effect of metallicity. The result is shown in Figure 7, which shows the data and following fit to the data:

$$
\begin{aligned}
\log _{10}\left(2 \theta_{*} / \mathrm{mas}\right)= & 0.53598+0.07427\left(V_{S 0}-H_{S 0}\right) \\
& +0.04511[\mathrm{Fe} / \mathrm{H}]-0.2 H_{S 0} .
\end{aligned}
$$

as shown in Figure 7. The subscripts so indicate extinctioncorrected source magnitudes. If we assume a $1.5 \%$ uncertainty in the model, $2.7 \%$ uncertainty from the $[\mathrm{Fe} / \mathrm{H}]$ error bar, $0.1 \mathrm{mag}$ uncertainty in $\left(V_{S 0}-H_{S 0}\right)$, and $0.02 \mathrm{mag}$ calibration uncertainty for $H_{S 0}$, then we find a $3.6 \%$ uncertainty for this relation. (Note that this does not include the light-curve model uncertainty in $H_{S 0}$, which will be handled by a different part of our analysis.)

Now that we have a formula for the angular source radius, $\theta_{*}$, we can determine that angular Einstein radius, $\theta_{E}=\theta_{*} t_{E} /$ $t_{*}$, for each light-curve model. This allows us to determine the lens mass, using Equation (2). The lens distance can also be determined using Equation (3), provided that the source distance, $D_{S}$, is known.

Table 2 gives the masses corresponding to the best-fit twoplanet and circumbinary models, which are $M_{L}=0.7185 M_{\odot}$ and $0.7835 M_{\odot}$, respectively, from Equation (2). If we assume a source distance of $8 \mathrm{kpc}$, Equation (3) indicates lens system distances of $D_{L}=2.96$ and $3.13 \mathrm{kpc}$, respectively.

\subsection{HST Images}

Shortly after the planetary signal was discovered in the OGLE-2007-BLG-349 light curve, an HST Director's Discretionary proposal was submitted to use the Wide Field Planetary Camera 2 (WFPC2) to observe this event. This proposal was approved as HST program GO/DD-11352. The approved program consisted of one short visit with a total of $320 \mathrm{~s}$ of exposures in the WFPC2 F814W passband on 2007 October 8, some 33 days after peak magnification, as well as one longer visit on 2008 May 4, 243 days after peak magnification. The longer visit included a total of $1280 \mathrm{~s}$ of exposures in each of the F555W and F814W passbands. The first visit occurred when the microlensing magnification was a factor of $A=$ 3.444 , and the magnification dropped to $A=1.036$ by the time of the second observation. Close-ups of summed images centered on the OGLE-2007-BLG-349 target from each visit are shown in Figure 8, and the change in magnification of the target is clearly visible. Because these images were taken within a year of peak magnification, the separation between the lens and source stars (Bennett et al. 2006, 2007, 2015) is not detectable.

These HST images were reduced by two independent reduction codes. The primary reduction used the reduction code of Anderson \& King (2000) and Anderson \& King (2004), calibrated to the OGLE-III database (Szymański et al. 2011), and the secondary reduction used HSTPHOT (Dolphin 2000a). The two reductions agree to better than $0.01 \mathrm{mag}$ in absolute calibration and better than 0.004 mag in the difference in magnitudes between the two epochs. However, these images were taken $\sim 14 \mathrm{yr}$ after the WFPC2 instrument was installed, and the WFPC2 detectors have suffered significant radiation damage during this time. This radiation damage has created defects in the detector that result in a significant reduction in the charge transfer efficiency (CTE) of the detectors. The effect of this CTE degradation is to reduce the sensitivity of the detectors, and we correct for this using the tool on the Space Telescope Science Institute website 


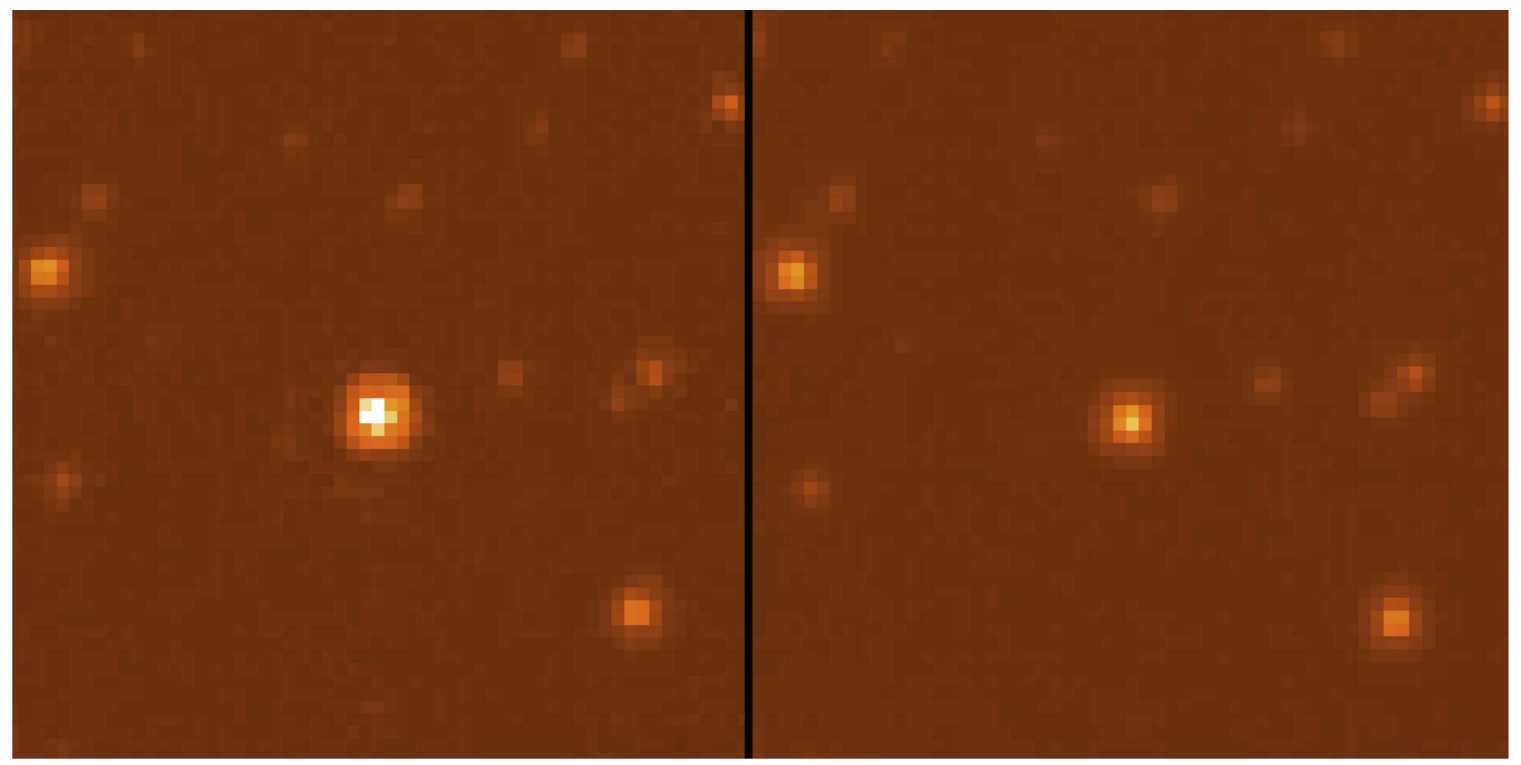

Figure 8. HST WFPC2 F814W images of the OGLE-2007-BLG-349 target, while magnified by a factor of $A=3.444$ in the left panel and nearly unmagnified by $A=$ 1.036 in the right panel.

(http://www.stsci.edu/hst/wfpc2/software/wfpc2_cte_calc1. html) based on the analysis of Dolphin (2000b). These corrections are magnitude dependent, so we have calculated the separate corrections for the lens-plus-source target and the brighter reference stars that are used to calibrate the HST images to the OGLE-III catalog (Szymański et al. 2011). For the F814W data, the Dolphin (2000b) code indicates target magnitude corrections of $-0.033 \mathrm{mag}$ for the first-epoch observations and -0.082 for the second-epoch observations, when the event had nearly ended. These corrected reductions give $I_{\mathrm{HST}}=18.930 \pm 0.004 \mathrm{mag}$ at $\mathrm{HJD}^{\prime}=4382.0353$ and $I_{\mathrm{HST}}=20.035 \pm 0.009 \mathrm{mag}$ at $\mathrm{HJD}^{\prime}=4590.7740 \equiv t_{H 2}$, where $\mathrm{HJD}^{\prime}=\mathrm{HJD}-2,450,000$. This later measurement of $I_{\mathrm{HST}}=$ $20.035 \pm 0.009 \mathrm{mag}$, at a magnification of $A=1.036$, is substantially brighter than the source magnitudes from the bestfit models presented in Table 2. It is substantially fainter than the combined source-plus-lens magnitude for the best twoplanet model $\left(I_{\mathrm{SL}}\left(t_{H 2}\right)=19.162\right)$, but it is very close to the combined source-plus-lens magnitude for the best-fit circumbinary model $\left(I_{\mathrm{SL}}\left(t_{H 2}\right)=20.009\right)$. (The details of how the lens -star magnitudes are estimated are discussed in the next section.) If the host star of the two-planet model was a white dwarf, then it would be extremely faint, and the lens-plussource brightness would be just the slightly magnified source at $I_{\mathrm{SL}}\left(t_{H 2}\right)=20.318$, which is substantially fainter than the $I$-band brightness measured in the HST images. This suggests that a circumbinary model is preferred, because a two-planet model with a main-sequence host would appear to be too bright to match the HST data, while a two-planet system orbiting a white dwarf would be too faint.

The WFPC2/F555W ( $V$-band) images can also constrain the lens system, and they also support the circumbinary model. The F555W images yield a CTE-corrected source-plus-lens magnitude of $V_{\mathrm{SL}}\left(t_{H 2}\right)=22.33 \pm 0.04$. This compares to predictions of $V_{\mathrm{SL}}\left(t_{H 2}\right)=21.38$ for the two-planet model with a mainsequence host and $V_{\mathrm{SL}}\left(t_{H 2}\right)=22.23$ for combined brightness of the source and two lens stars for the circumbinary model. So, the HST V-band data seem to clearly favor the circumbinary model, as well. However, if the planetary host star was a white dwarf, the host star brightness would be negligible, so it would have $V_{\mathrm{SL}}\left(t_{H 2}\right)=22.30$. This is consistent with the HST V-band measurement.

These comparisons between the best-fit two-planet and circumbinary models and the HST data suggest that the circumbinary model is favored, but to reach a firm conclusion, we need to consider more than the best-fit models. We must determine which models are consistent with both the lightcurve data and the HST images. The F814W (I-band) images provide a much stronger constraint than the F555W ( $V$-band) images, because the low-mass lens stars are brighter in the $I$ band and because the uncertainties in both the extinction and CTE correction are larger in the $V$ band. In the next section, we will apply a constraint from the HST F814W observations to the light-curve models and find the light-curve models in each category that are most consistent with the light curve and HST F814W images. We will also compare these constrained models with the HST F555W data.

\subsection{Light-curve Models with HST Constraint}

In order to determine which of our models are consistent with the HST imaging data, we perform a set of constrained fits in which the lens system is forced to match the HST observations. We consider three different possibilities:

1. Two-planet, one-star model with a single main-sequence host star.

2. Two-planet, one-star model with a single white dwarf host star of negligible brightness.

3. One-planet, two-star model with a circumbinary planet orbiting a pair of main-sequence stars.

In principle, we could also consider circumbinary planets orbiting a binary consisting of at least one white dwarf, but the primary goal of this exercise is to establish that this is, in fact, a circumbinary planet. Also, white dwarfs generally form at a late stage of stellar evolution, after earlier stages of stellar evolution that may have removed planets from the vicinity of the Einstein ring, where they are detectable by microlensing. 
(Mass loss by stars on the giant or supergiant branch or during planetary nebula formation could shift planets to wide orbits or unbind them from their former host star, depending on the details of the mass-loss processes.)

At a Galactic latitude of $b=-2.5161$ and a lens distance of $\sim 3 \mathrm{kpc}$, the lens system is likely to be behind about $3 / 4$ of the dust that is in the foreground of the source. We model the dust with a simple exponential scale height of $h_{\text {dust }}=0.10 \pm 0.02 \mathrm{kpc}$ (Drimmel \& Spergel 2001), so that the extinction in the foreground of the lens is given by

$$
A_{i, L}=\frac{1-e^{-\left|D_{L} /\left(h_{\mathrm{dust}} \sin b\right)\right|}}{1-e^{-\left|D_{S} /\left(h_{\mathrm{dust}} \sin b\right)\right|}} A_{i, S},
$$

where the index $i$ refers to the passband, which is the $I$ band in this case.

For possibility 2, a two-planet model with a white dwarf host, all the detectable flux comes from the source star, which is directly determined by the fit. So, the uncertainty in the extinction plays no role. Therefore, for these models we constrain the very slightly lensed source brightness at the time of the second-epoch HST observation $\left(\mathrm{HJD}^{\prime}=4590.7740\right)$ to be $I=20.035 \pm 0.010$.

For possibilities 1 and 3, we require a mass-luminosity relation, and we use the same empirical mass-luminosity relation that was used in Bennett et al. (2015). We use the mass-luminosity relations of Henry \& McCarthy (1993), Henry et al. (1999), and Delfosse et al. (2000) in different mass ranges. For $M_{L}>0.66 M_{\odot}$, we use the Henry \& McCarthy (1993) relation; for $0.12 M_{\odot}<M_{L}<0.54 M_{\odot}$, we use the Delfosse et al. (2000) relation; and for $0.07 M_{\odot}<M_{L}<0.10 M_{\odot}$, we use the Henry et al. (1999) relation. In between these mass ranges, we linearly interpolate between the two relations used on the boundaries. That is, we interpolate between the Henry \& McCarthy (1993) and the Delfosse et al. (2000) relations for $0.54 M_{\odot}<M_{L}<0.66 M_{\odot}$, and we interpolate between the Delfosse et al. (2000) and Henry et al. (1999) relations for $0.10 M_{\odot}<M_{L}<0.12 M_{\odot}$.

The extinction is also an important uncertainty for possibilities 1 and 3. We use Equation (5) to estimate the extinction, but we also need to include a reasonable uncertainty for this model. About one-third of the flux at the second-epoch observation (at $\mathrm{HJD}^{\prime}=4590.7740$ ) is due to the lens, so an $11 \%$ uncertainty in the extinction in the foreground of the lens would correspond to a $3.7 \%$ uncertainty in the combined lens-plus-source flux, or a 0.04 mag uncertainty when combined with the 0.01 mag uncertainty assumed for the HST calibration. We therefore apply the constraint $I_{\mathrm{SL}}\left(t_{H 2}\right)=20.035 \pm 0.040$ on the combined source-plus-lens flux at $\mathrm{HJD}^{\prime}=t_{H 2}=4590.7740$.

For the circumbinary models, the fit parameters (along with $\theta_{*}$ ) determine the source distance, if we insist that the stellar orbits be circular. (The modeling employs circular orbits, but these can be interpreted as second-order approximations to any bound orbit. See Bennett et al. [2010] for more discussion of this point.) With $\theta_{E}$ and $\pi_{E}$ determined, we know the mass of the lens system, via Equation (2), and we also know the five parameters describing the orbit, $d_{23}, \phi_{23}, \dot{d}_{23 x}, \dot{d}_{23 y}$, and $1 / T_{\text {orb }}$ in Einstein radius units. We only need the distance to the lens system to convert these to physical units, and this is given by Equation (3) (assuming that we already know $D_{S}$ ). However, we already know the size of the orbit in physical units, via Kepler's third law, since we know the period and the mass of the binary host system. So, we can use this information to invert Equation (3) and solve for the source distance. From the CMD in Figure 6, we see that the source lies on the red side of the bulge main sequence, and we know that the red color is explained by the high metallicity measured by Bensby et al. (2013). So, it is safe to assume that the source is located in the bulge. We therefore apply a constraint on the implied distance to the source in the circumbinary models, $D_{S}=7.8 \pm 1.4 \mathrm{kpc}$, assuming the bulge distance estimate by Nataf et al. (2013) at the Galactic longitude of this event.

Table 3 gives the parameters of the best-fit models with the source-plus-lens $I$-band magnitude $\left(I_{\mathrm{SL}}\right)$ constraint imposed at the time, $t_{H 2}$, of the second epoch of HST observations. The best-fit circumbinary model does very well with the constraint on the $I$-band lens-plus-source brightness, as the application of this constraint only increases $\chi^{2}$ by $\Delta \chi^{2}=0.18$ for one additional degree of freedom. The two-planet models are so significantly disfavored that we can exclude them based on these constrained fits. The best two-planet model with a mainsequence host is disfavored by $\Delta \chi^{2}=56.45$ with respect to the best circumbinary model, and the two-planet model with a dark stellar remnant host is disfavored by $\Delta \chi^{2}=43.76$. For the main-sequence host case, $\Delta \chi^{2}=33.13$ comes from the $I_{\mathrm{SL}}\left(t_{H 2}\right)$ constraint and $\Delta \chi^{2}=23.32$ comes from the difference in the light-curve model fits. In the case of a dark stellar remnant host, almost the entire $\Delta \chi^{2}$ difference comes from the light-curve difference. These $\chi^{2}$ differences are sufficient to exclude both the two-planet and white dwarf host models. If we assume Gaussian random errors, then the probability of the best non-circumbinary solution is $3 \times 10^{-9}$. A very conservative choice would be to substitute $\Delta \chi^{2} / 2$ for $\Delta \chi^{2}$ into the $\chi^{2}$ probability distribution formula. This is equivalent to assuming that the correlations and non-Gaussianity of the errors have the same effect as increasing each error bar (and constraint) by a factor of $\sqrt{2}$. With this assumption, the probability of the best non-circumbinary model would be $2 \times 10^{-5}$, so even with a very conservative assumption about the effects of nonGaussian and correlated errors, it is only the circumbinary planetary models that are viable. We also note that the circumbinary models can be confirmed by observing the lens stars with the predicted brightness of $I_{L}=21.39 \pm 0.24$ and $H_{L}=18.57 \pm 0.22$ separating from the source at the predicted rate of $\mu_{\text {rel }}=3.55 \pm 0.15$ mas yr$^{-1}$. (These numbers come from the MCMC calculations discussed later in this section.)

We can also compare the measured V-band HST (F555W) magnitudes to the predicted values from these constrained models. The best-fit constrained circumbinary model predicts $V_{\mathrm{SL}}\left(t_{H 2}\right)=22.26 \pm 0.07$, which compares to the measured value of $V_{\mathrm{SL}}\left(t_{H 2}\right)=22.33 \pm 0.04$ for $\Delta \chi^{2}=0.75$. The twoplanet models do not do as well. With a main-sequence host star, we have the prediction of $V_{\mathrm{SL}}\left(t_{H 2}\right)=22.11 \pm 0.07$, which is still too bright and implies $\Delta \chi^{2}=7.44$. For a white dwarf host, which matched the HST $V$-band measurement without the HST I-band constraint, the constraint has pushed the $V$-band magnitude to be too bright, $V_{\mathrm{SL}}\left(t_{H 2}\right)=22.13 \pm 0.01$. Comparison to the measurement yields $\Delta \chi^{2}=23.53$, so it is only the circumbinary model that is consistent with the HST V-band measurement.

This comparison with HST images also rules out the models in which the planet orbits one member of a wide binary star system, although these were already excluded due to the lack of an acceptable light-curve fit. The microlensing parallax measurement constrains the mass interior to the Einstein radius 
Table 3

Best-fit Nonlinear Model Parameters with HST I-band Flux Constraint

\begin{tabular}{|c|c|c|c|c|c|c|c|}
\hline \multirow[b]{3}{*}{ Param. } & \multirow[b]{3}{*}{ Units } & & & \multicolumn{4}{|c|}{ Circumbinary } \\
\hline & & \multicolumn{2}{|c|}{ Two-planet } & \multicolumn{2}{|c|}{$u_{0}<0$} & \multicolumn{2}{|c|}{$u_{0}>0$} \\
\hline & & MS & WD & $d_{1 \mathrm{~cm}}<1$ & $d_{1 \mathrm{~cm}}>1$ & $d_{1 \mathrm{~cm}}<1$ & $d_{1 \mathrm{~cm}}>1$ \\
\hline$t_{E}$ & days & 112.758 & 95.738 & 117.793 & 121.141 & 118.939 & 120.191 \\
\hline$t_{0}$ & $\mathrm{HJD}^{\prime}$ & 4348.7472 & 4348.7467 & 4348.7465 & 4348.7520 & 4348.7459 & 4348.7511 \\
\hline$u_{0}$ & $\ldots$ & 0.002072 & 0.002443 & -0.001981 & -0.002051 & 0.001966 & 0.002077 \\
\hline$d_{1 \mathrm{~cm}}$ & $\cdots$ & 0.79580 & 0.79647 & 0.81424 & 1.22544 & 0.81399 & 1.22632 \\
\hline$d_{23}$ & $\ldots$ & 1.05349 & 0.95080 & 0.01903 & 0.01951 & 0.01784 & 0.01801 \\
\hline$\theta_{1 \mathrm{~cm}}$ & $\mathrm{rad}$ & 1.89675 & 1.89351 & 4.35929 & 4.35886 & 1.91859 & 1.91626 \\
\hline$\phi_{23}$ & $\mathrm{rad}$ & -3.07236 & -3.07866 & 0.37073 & 0.35840 & -0.40457 & -0.42858 \\
\hline$\epsilon_{1}$ & $10^{-4}$ & 3.7893 & 4.4687 & 3.4119 & 3.3134 & 3.3895 & 3.3471 \\
\hline$\epsilon_{2}$ & $\ldots$ & $9.304 \times 10^{-6}$ & $9.827 \times 10^{-6}$ & 0.45967 & 0.42723 & 0.48342 & 0.48489 \\
\hline$\epsilon_{3}$ & $\ldots$ & 0.99961 & 0.99954 & 0.53999 & 0.57244 & 0.51624 & 0.51477 \\
\hline$t_{*}$ & days & 0.06954 & 0.06929 & 0.07048 & 0.07072 & 0.07074 & 0.07056 \\
\hline$\dot{d}_{23 x}$ & day $^{-1}$ & 0.0 & 0.0 & 0.010586 & 0.012073 & 0.008882 & 0.009545 \\
\hline$\dot{d}_{23 y}$ & day $^{-1}$ & 0.0 & 0.0 & -0.006420 & -0.006506 & 0.005603 & 0.003518 \\
\hline $1 / T_{\text {orb }}$ & day $^{-1}$ & 0.0 & 0.0 & 0.060459 & 0.070847 & 0.059174 & 0.059632 \\
\hline$\pi_{E}$ & $\ldots$ & 0.36051 & 0.19544 & 0.18361 & 0.20926 & 0.13819 & 0.14066 \\
\hline$\phi_{E}$ & $\mathrm{rad}$ & 2.81614 & 2.38046 & 0.59559 & 0.48368 & 0.79651 & 0.76876 \\
\hline$\theta_{E}$ & mas & 1.1082 & 1.0261 & 1.1167 & 1.1282 & 1.1184 & 1.1273 \\
\hline$M_{L}$ & $M_{\odot}$ & 0.3775 & 0.6447 & 0.7468 & 0.6620 & 0.9937 & 0.9841 \\
\hline$I_{S c}$ & mag & 20.298 & 20.138 & 20.365 & 20.396 & 20.375 & 20.386 \\
\hline$I_{S h}$ & mag & 20.317 & 20.072 & 20.371 & 20.409 & 20.389 & 20.403 \\
\hline$I_{L}$ & mag & 21.006 & $\ldots$ & 21.527 & 21.484 & 21.410 & 21.390 \\
\hline$I_{\mathrm{SL}}\left(t_{H 2}\right)$ & mag & 19.826 & 20.053 & 20.020 & 20.036 & 20.001 & 20.005 \\
\hline$V_{S}$ & mag & 22.328 & 22.148 & 22.375 & 22.406 & 22.385 & 22.396 \\
\hline$H_{S}$ & mag & 18.219 & 18.040 & 18.268 & 18.267 & 18.277 & 18.288 \\
\hline fit $\chi^{2}$ & $\ldots$ & 3438.88 & 3426.19 & 3382.43 & 3387.85 & 3408.17 & 3412.62 \\
\hline dof & $\ldots$ & 3569 & 3569 & 3567 & 3567 & 3567 & 3567 \\
\hline
\end{tabular}

Note. $\mathrm{HJD}^{\prime}=\mathrm{HJD}-2,450,000$. The best fit parameters are indicated in bold face.

of the primary lens mass. This is the single host star of the twoplanet models or both host stars for a circumbinary system. For the case of a planet orbiting one star of a wide binary system, the mass constrained by the microlensing parallax measurement is the mass of the planetary host star. Its wide binary companion just provides a small perturbation to the light curve. So, these models are also excluded by the same argument that excludes the two-planet models. The microlensing parallax measurement requires a planetary host mass of $\sim 0.7 M_{\odot}$, and it is only if the host is a close binary system that this mass can be split into two stars that are consistent with the HST images.

For the circumbinary models, we present the best-fit model parameters for each of the degenerate solutions, with $u_{0}<0$ or $u_{0}>0$ and with $d_{1 \mathrm{~cm}}<1$ or $d_{1 \mathrm{~cm}}>1$, in Table 3 . The $u_{0}>0$ models have smaller parallaxes and therefore larger host star masses, and this means that they are disfavored by the $I_{\mathrm{SL}}\left(t_{H_{2}}\right)$ constraint by $\Delta \chi^{2} \sim 26-30$. The wide models with $d_{1 \mathrm{~cm}} \approx 1.225$ are also slightly disfavored by $\Delta \chi^{2} \sim 5$.

In order to determine the ranges of parameters and properties that are consistent with the observed light curve and HST constraint, we have performed a series of Markov Chain Monte Carlo (MCMC; Verde et al. 2003) runs. We follow the usual procedure (Bennett et al. 2008) of weighting each class of models with the weight function, $e^{-\Delta \chi^{2} / 2}$, where $\Delta \chi^{2}$ refers to the $\chi^{2}$ difference between the local $\chi^{2}$ minimum and the global $\chi^{2}$ minimum (with $u_{0}<0$ and $d_{1 \mathrm{~cm}}<1$ ). For the $u_{0}<0$,
Table 4

Constrained Circumbinary MCMC Model Parameters

\begin{tabular}{|c|c|c|}
\hline Parameter & Units & Mean MCMC Values \\
\hline$t_{E}$ & days & $118 \pm 4$ \\
\hline$t_{0}$ & $\mathrm{HJD}^{\prime}$ & $4348.7470 \pm 0.0014$ \\
\hline$u_{0}$ & $\cdots$ & $-0.00198 \pm 0.00007$ \\
\hline$d_{1 \mathrm{~cm}}$ & close mod. & $0.8146 \pm 0.0015$ \\
\hline$\left(d_{1 \mathrm{~cm}}\right)$ & wide mod. & $1.2257 \pm 0.0022$ \\
\hline$d_{23}$ & $\cdots$ & $0.0193 \pm 0.0010$ \\
\hline$\theta_{1 \mathrm{~cm}}$ & radians & $4.3590 \pm 0.0030$ \\
\hline$\phi_{23}$ & radians & $0.368 \pm 0.056$ \\
\hline$\epsilon_{1}$ & $\cdots$ & $(3.39 \pm 0.12) \times 10^{-4}$ \\
\hline$\epsilon_{2}$ & $\cdots$ & $0.493 \pm 0.098$ \\
\hline$\epsilon_{3}$ & $\cdots$ & $0.507 \pm 0.098$ \\
\hline$t_{*}$ & days & $0.07065 \pm 0.00043$ \\
\hline$\dot{d}_{12 x}$ & day $^{-1}$ & $0.0096 \pm 0.0023$ \\
\hline$\dot{d}_{12 y}$ & day $^{-1}$ & $-0.0069 \pm 0.0043$ \\
\hline $1 / T_{\text {orb }}$ & day $^{-1}$ & $0.061 \pm 0.091$ \\
\hline$\pi_{E}$ & $\cdots$ & $0.204 \pm 0.034$ \\
\hline$\phi_{E}$ & radians & $0.54 \pm 0.11$ \\
\hline$\mu_{\text {rel }}$ & $\operatorname{mas} \mathrm{yr}^{-1}$ & $3.55 \pm 0.15$ \\
\hline$\theta_{E}$ & mas & $1.15 \pm 0.05$ \\
\hline$V_{S}$ & mag & $22.380 \pm 0.037$ \\
\hline$I_{S}$ & mag & $20.369 \pm 0.037$ \\
\hline$H_{S}$ & mag & $18.272 \pm 0.037$ \\
\hline
\end{tabular}

Note. HJD $^{\prime}=$ HJD-2,450,000. The close model is preferred over the wide model by $\Delta \chi^{2}=4.92$. 
Table 5

Physical Parameters

\begin{tabular}{llcc}
\hline \hline Parameter & Units & Average Value & $2 \sigma$ Range \\
\hline$D_{L}$ & $\mathrm{kpc}$ & $2.76 \pm 0.38$ & $2.06-3.56$ \\
$M_{\mathrm{A}+\mathrm{B}}$ & $M_{\odot}$ & $0.71 \pm 0.12$ & $0.50-0.95$ \\
$M_{\mathrm{A}}$ & $M_{\odot}$ & $0.41 \pm 0.07$ & $0.28-0.54$ \\
$M_{\mathrm{B}}$ & $M_{\odot}$ & $0.30 \pm 0.07$ & $0.15-0.45$ \\
$m_{c}$ & $M_{\oplus}$ & $80 \pm 13$ & $56-107$ \\
$a_{\perp \mathrm{AB}}$ & $\mathrm{au}$ & $0.061 \pm 0.007$ & $0.048-0.074$ \\
$a_{\mathrm{AB}}$ & $\mathrm{au}$ & $0.080_{-0.015}^{+0.027}$ & $0.054-0.162$ \\
$P_{\mathrm{AB}}$ & days & $9.7_{-2.5}^{+5.5}$ & $5.7-28.1$ \\
$a_{\perp \mathrm{CM} c}$ & $\mathrm{au}$ & $2.59_{-0.34}^{+0.43}$ & $1.97-3.89$ \\
$V_{L}$ & $\mathrm{mag}$ & $24.73 \pm 0.34$ & $24.18-25.51$ \\
$I_{L}$ & $\mathrm{mag}$ & $21.39 \pm 0.24$ & $20.98-21.98$ \\
$H_{L}$ & $\mathrm{mag}$ & $18.57 \pm 0.22$ & $18.22-19.07$ \\
\hline
\end{tabular}

Note. The average value is the mean value for all parameters except that we use the median for $a_{\perp \mathrm{AB}}, a_{\mathrm{AB}}, P_{\mathrm{AB}}$, and $a_{\perp \mathrm{CM} c}$.

$d_{1 \mathrm{~cm}}>1$ models, the penalty is $\Delta \chi^{2}=5.42$, which corresponds to a weight of 0.067 , but for the $u_{0}>0$ models the penalties are $\Delta \chi^{2}=25.74$ (for $d_{1 \mathrm{~cm}}<1$ ) and $\Delta \chi^{2}=30.19$ (for $d_{1 \mathrm{~cm}}>1$ ), corresponding to weights of $3 \times 10^{-6}$ and $3 \times 10^{-7}$, respectively. The weights for the $u_{0}>0$ models are so small that they do not contribute to the mean microlens model parameters, shown in Table 4 . The physical parameters of the lens system from these MCMC runs are given in Table 5. The system consists of a planet of $80 \pm 13 M_{\oplus}$ orbiting a binary stellar system, consisting of two $\mathrm{M}$ dwarfs with masses of $0.41 \pm 0.07 M_{\odot}$ and $0.30 \pm 0.07 M_{\odot}$. These stars have a semimajor axis of $0.080_{-0.015}^{+0.027}$ au and a period of $9.7_{-2.5}^{+5.4}$ days. The median two-dimensional separation of the planet from the stellar center of mass is $2.59_{-0.34}^{+0.43}$ au, which implies a median semimajor axis of $\sim 3.2 \mathrm{au}$ and an orbital period of about $7 \mathrm{yr}$ if we assume a random orbital orientation. However, it is only the transverse separation that is measured (and reported in Table 5), so the three-dimensional separation could be much larger if the line-of-sight separation between the planet and binary stars is large. Then, the semimajor axis and orbital period could be substantially larger than this.

\section{VERY LARGE TELESCOPE (VLT)/NACO OBSERVATIONS OF THE SOURCE-PLUS-LENS SYSTEM}

We have also obtained two epochs of adaptive optics observations in the infrared $J H K$ passbands with the VLT NACO instrument with a $28^{\prime \prime} \times 28^{\prime \prime}$ field of view (FOV). The first $H$-band observations were taken at $\mathrm{HJD}^{\prime}=4386.046880$, when the magnification was about a factor of 3 , and the secondepoch observations were taken at $\mathrm{HJD}^{\prime}=4686.144531$, when the magnification was only about $1 \%$. This small FOV made calibration of the VLT/NACO data very difficult, and, in fact, we were unable to find a satisfactory calibration of these data. We were able to calibrate the CTIO $\mathrm{H}$-band data as discussed in Section 4.1, and so from the CTIO $H$-band light curve, we know the $H$-band source magnitude.

Each of the different models presented in Table 3 has a different prediction for the combined lens-plus-source magnitude at the times of the two different observations. The observations give a magnitude difference of $0.87 \pm 0.10$ between the two epochs. This compares to the $0.575,1.010$, and 0.680 source-plus-lens $H$-band magnitude difference

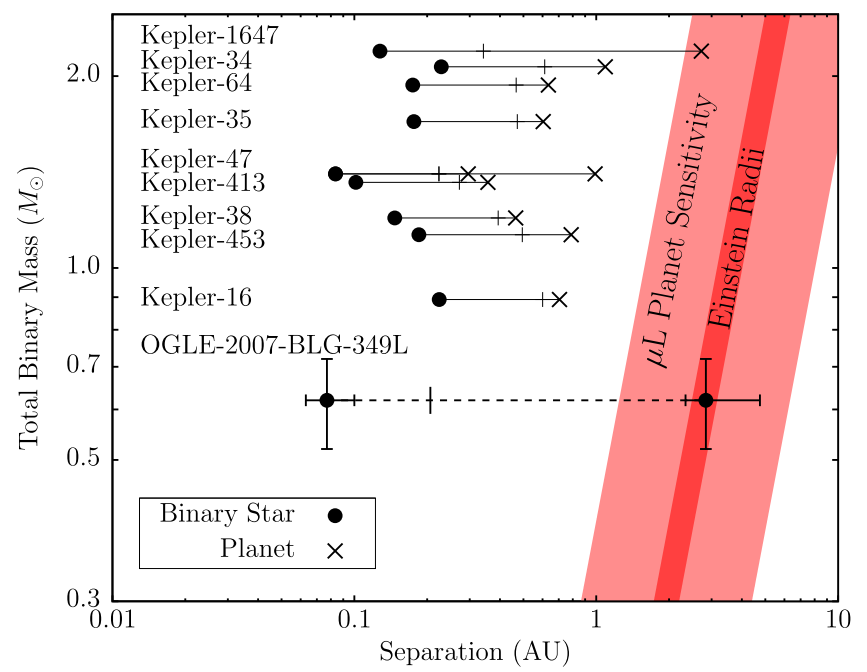

Figure 9. Comparison of host star masses and orbital separations for the known circumbinary planet systems. The filled circles show the orbital separations of the host stars, while the orbital separations of the planets from the stellar centers of mass are marked with crosses. The vertical bars on each line indicate the approximate stability limit. The red region gives the typical Einstein radius as a function of mass, and the light-red region gives the approximate range of planetary microlens sensitivity.

between the first and second epochs for the two-planet plus main-sequence host, two-planet plus stellar remnant host, and circumbinary models, respectively. These differ from the measured value by $2.95 \sigma, 1.40 \sigma$, and $1.90 \sigma$, respectively. So, the VLT/NACO data slightly favor the circumbinary model over the two-planet model with a main-sequence host, but a white dwarf host does slightly better. The effect is too small to alter our conclusions, however.

\section{DISCUSSION AND CONCLUSIONS}

In the previous section, we have established that although the OGLE-2007-BLG-349 light curve can be explained by models with one star and two planets, it is only the circumbinary planet models that can explain both the light curve and the HST observations. So, the system consists of two host stars, OGLE2007-BLG-349LA and OGLE-2007-BLG-349LB, orbited by a planet somewhat less massive than Saturn. Although it was the first circumbinary planet to be observed, aside from a planet orbiting a neutron star-white dwarf system (Ford et al. 2000; Sigurdsson et al. 2003), it was not the first circumbinary planet to be published, as 10 circumbinary planets (Doyle et al. 2011; Welsh et al. 2015; Kostov et al. 2016) have been discovered by the Kepler mission.

One puzzle with the circumbinary planets discovered in the Kepler data is that most of them are located quite close to the stability limit (Holman \& Wiegert 1999), as shown in Figure 9. That is, if they were moved to orbits with slightly smaller semimajor axes, they would quickly become dynamically unstable. Holman \& Wiegert (1999) find that circular, coplanar circumbinary orbits become unstable within $a_{c} \simeq(2.28 \pm$ $0.01)+(3.8 \pm 0.3) e+(1.7 \pm 0.1) e^{2}$, where $e$ is the eccentricity of the binary orbit and $a_{c}$ is measured in units of the stellar binary semimajor axis. Our modeling has enforced a circular orbit for the stellar binary, so it is sensible to assume a low eccentricity. Also, if the stellar binary orbit does have a significant eccentricity, then it is likely that the semimajor axis 
is smaller than the mean values listed in Table 5, because stars spend most of their time in an eccentric orbit at separations larger than the semimajor axis. So, the consideration of stellar binary orbits with significant eccentricity is not likely to significantly increase the maximum stellar separation, which is closely related to the stability constraint. So, we assume $e \approx 0.1$, and this yields $a_{c}=2.7$. The median semimajor axis of the OGLE-2007-BLG-349LAB binary, $a \simeq 0.080 \mathrm{au}$ from Table 5, and the median three-dimensional separation between stellar center-of-mass and the OGLE-2007-BLG-349L(AB)c planet is $\sim 3.2 \mathrm{au}$. Therefore, we estimate the planet orbits at $\sim 15 a_{c}$. This compares to most of the Kepler circumbinary planets that orbit at $<2 a_{c}$ and the widest-orbit Kepler circumbinary planet (Kostov et al. 2016) that orbits at $7 a_{c}$.

The expected orbital period for the OGLE-2007-BLG-349L (AB)c planet is $\sim 7 \mathrm{yr}$ assuming a host system mass of $0.71 M_{\odot}$ and a semimajor axis of 3.2 au, so such a system could not have been detected by Kepler. The only Kepler planet with a comparable separation is Kepler-1647b. It orbits a star system that is three times more massive than the OGLE-2007-BLG349L host star system, which implies a period short enough to allow for its detection with two transit episodes during Kepler observations. We expect that Kepler's detection efficiency for such systems is quite low, so such systems might be quite common.

The fact that the first circumbinary planet found by microlensing has an orbital separation well beyond the stability limit adds modest support to the idea that circumbinary planets far beyond the stability limit are quite common. This would imply that circumbinary planets probably form in the outer disk, relatively far from the orbital stability limit (Kley \& Haghighipour 2014; Bromley \& Kenyon 2015; Silsbee \& Rafikov 2015) instead of in situ (Meschiari 2014). In principle, this new microlensing discovery could provide strong evidence that circumbinary planets are substantially more common far from the stability limit than close to the stability limit (Luhn et al. 2016). Microlensing is most sensitive to both planets and stellar companions at separations close to the Einstein radius. However, for event OGLE-2007-BLG-349, the ratio of the two-dimensional separation between the planet and stellar center of mass to the separation between the two stars is 42 . Such a large ratio was only detectable because of the very high magnification of this event, but circumbinary planets with a smaller separation ratio should be detectable for a much larger class of lower-magnification events. The fact that no other circumbinary planets have been found by microlensing might be considered to imply that circumbinary planets with smaller separation ratios are more rare. However, there is circumstantial evidence suggesting that we may be inefficient at identifying such events in our data. Unlike the transit method, microlensing is sensitive to planets beyond the snow line, (Lecar 2006; Kennedy \& Kenyon 2008), so OGLE-2007-BLG$349 \mathrm{~L}(\mathrm{AB}) \mathrm{c}$ is the first circumbinary planet beyond the snow line.

Gould et al. (2014) presented another two-star-plus-oneplanet event, OGLE-2013-BLG-0341, which was interpreted as a wide binary with a planet orbiting one of the two stars, although there are circumbinary models with very similar light curves. This was also a high-magnification event with the signal dominated by the stellar binary instead of by the planet (like OGLE-2007-BLG-349). However, the lens-source alignment was such that the source crossed a planetary caustic feature prior to reaching high magnification. This made it obvious that the lens system included a planet, but we were very lucky to have this planetary feature detected. And the analysis showed that the planet was required to fit the data even if the low-magnification planetary feature was not seen. This suggests that there should be many more two-star-plus-oneplanet events in the data that we have already collected, but that we are not efficient at finding planetary signals in events that are dominated by stellar binary microlensing features. Hence, we recommend a systematic search for planetary signals in the light curves of strong stellar binary events. If a large population of circumbinary planets are found, it will add to the $\sim 10 \%$ frequency of circumbinary planets found in short-period orbits (Armstrong et al. 2014). Circumbinary planetary systems can be quite efficient at ejecting planets (Smullen et al. 2016; Sutherland \& Fabrycky 2016), so they could contribute to the large population of rogue planets found by microlensing (Sumi et al. 2011).

The authors would like to thank Subo Dong for a great deal of work on this event, including the initial realization that a third mass was needed to fit the light curve. D.P.B., A.B., and D.S. were supported by NASA through grants NASANNX12AF54G and NNX13AF64G. The MOA group acknowledges financial support from the Marsden Fund of NZ and in Japan from grants JSPS25103508 and 23340064. T. S. received support from JSPS23103002, JSPS24253004, and JSPS26247023. A.G. and B.S.G. were supported by NSF grant AST-1516842. The OGLE Team thanks Profs. M. Kubiak and G. Pietrzyński, former members of the OGLE team, for their contribution to the collection of the OGLE photometric data over the past years. The OGLE project has received funding from the National Science Centre, Poland, grant MAESTRO 2014/14/A/ST9/00121 to A.U. NASA/ESA Hubble Space Telescope data from program GO/DD-11352 were obtained from the data archive at the Space Telescope Science Institute. STScI is operated by the Association of Universities for Research in Astronomy, Inc., under NASA contract NAS 5-26555. Work by C.H. was supported by the Creative Research Initiative Program (2009-0081561) of the National Research Foundation of Korea. K.H. acknowledges support from STFC grant ST/M001296/1.

\section{REFERENCES}

Adams, A., Boyajian, T. S., \& von Braun, K. 2016, in American Astronomical Society Meeting Abstracts 227, \#138.04

Alard, C., \& Lupton, R. H. 1998, ApJ, 503, 325

Albrow, M. D. 2000, ApJ, 534, 894

Albrow, M. D., Horne, K., Bramich, D. M., et al. 2009, MNRAS, 397, 2099

Alcock, C., Allsman, R. A., Alves, D., et al. 1995, ApJL, 454, L125

An, J. H., Albrow, M. D., Beaulieu, J.-P., et al. 2002, ApJ, 572, 521

Anderson, J., \& King, I. R. 2000, PASP, 112, 1360

Anderson, J., \& King, I. R. 2004, Hubble Space Telescope Advanced Camera for Surveys Instrument Science Report 04-15

Armstrong, D. J., Osborn, H. P., Brown, D. J. A., et al. 2014, MNRAS, 444, 1873

Bennett, D. P. 2008, in Exoplanets, ed. J. Mason (Berlin: Springer) arXiv:0902.1761)

Bennett, D. P. 2010, ApJ, 716, 1408

Bennett, D. P., Alcock, C., Allsman, R., et al. 1993, BAAS, 25, 1402

Bennett, D. P., Anderson, J., Bond, I. A., Udalski, A., \& Gould, A. 2006, ApJL, 647, L171

Bennett, D. P., Anderson, J., \& Gaudi, B. S. 2007, ApJ, 660, 781

Bennett, D. P., Bhattacharya, A., Anderson, J., et al. 2015, ApJ, 808, 169

Bennett, D. P., Bond, I. A., Udalski, A., et al. 2008, ApJ, 684, 663

Bennett, D. P., \& Rhie, S. H. 1996, ApJ, 472, 660 
Bennett, D. P., Rhie, S. H., Becker, A. C., et al. 1999, Natur, 402, 57 Bennett, D. P., Rhie, S. H., Nikolaev, S., et al. 2010, ApJ, 713, 837 Bensby, T., Yee, J. C., Feltzing, S., et al. 2013, A\&A, 549, A147 Bond, I. A., Abe, F., Dodd, R. J., et al. 2001, MNRAS, 327, 868 Boyajian, T. S., van Belle, G., \& von Braun, K. 2014, AJ, 147, 47 Bramich, D. M. 2008, MNRAS, 386, L77

Bromley, B. C., \& Kenyon, S. J. 2015, ApJ, 806, 98

Cardelli, J. A., Clayton, G. C., \& Mathis, J. S. 1989, ApJ, 345, 245

Carpenter, J. M. 2001, AJ, 121, 2851

Cohen, J. G., Huang, W., Udalski, A., Gould, A., \& Johnson, J. A. 2008, ApJ, 682, 1029

Delfosse, X., Forveille, T., Ségransan, D., et al. 2000, A\&A, 364, 217

Dolphin, A. E. 2000a, PASP, 112, 1383

Dolphin, A. E. 2000b, PASP, 112, 1397

Dong, S., Bond, I. A., Gould, A., et al. 2009, ApJ, 698, 1826

Dong, S., DePoy, D. L., Gaudi, B. S., et al. 2006, ApJ, 642, 842

Doyle, L. R., Carter, J. A., Fabrycky, D. C., et al. 2011, Sci, 333, 1602

Drimmel, R., \& Spergel, D. N. 2001, ApJ, 556, 181

Ford, E. B., Joshi, K. J., Rasio, F. A., \& Zbarsky, B. 2000, ApJ, 528, 336

Gaudi, B. S. 2012, ARA\&A, 50, 411

Gaudi, B. S., Bennett, D. P., Udalski, A., et al. 2008, Sci, 319, 927

Gould, A. 1992, ApJ, 392, 442

Gould, A. 2014, JKAS, 47, 215

Gould, A., Udalski, A., Monard, B., et al. 2009, ApJL, 698, L147

Gould, A., Udalski, A., Shin, I.-G., et al. 2014, Sci, 345, 46

Han, C., Bennett, D. P., Udalski, A., et al. 2016, ApJ, 825, 8

Henry, T. J., Franz, O. G., Wasserman, L. H., et al. 1999, ApJ, 512, 864

Henry, T. J., \& McCarthy, D. W., Jr. 1993, AJ, 106, 773

Holman, M. J., \& Wiegert, P. A. 1999, AJ, 117, 621

Holtzman, J. A., Watson, A. M., Baum, W. A., et al. 1998, AJ, 115, 1946

Jung, Y. K., Han, C., Gould, A., \& Maoz, D. 2013, ApJL, 768, L7

Kennedy, G. M., \& Kenyon, S. J. 2008, ApJ, 673, 502

Kennedy, G. M., Kenyon, S. J., \& Bromley, B. C. 2006, ApJL, 650, L139
Kervella, P., Thévenin, F., Di Folco, E., \& Ségransan, D. 2004, A\&A, 426, 297

Kley, W., \& Haghighipour, N. 2014, A\&A, 564, A72

Kostov, V. B., McCullough, P. R., Carter, J. A., et al. 2014, ApJ, 784, 14

Kostov, V. B., McCullough, P. R., Hinse, T. C., et al. 2013, ApJ, 770, 52

Kostov, V. B., Orosz, J. A., Welsh, W. F., et al. 2016, ApJ, 827, 86

Lecar, M., Podolak, M., Sasselov, D., \& Chiang, E. 2006, ApJ, 640, 1115

Lissauer, J. J., Fabrycky, D. C., Ford, E. B., et al. 2011, Natur, 470, 53

Luhn, J. K., Penny, M. T., \& Gaudi, B. S. 2016, ApJ, 827, 61

Mayor, M., \& Queloz, D. 1995, Natur, 378, 355

Meschiari, S. 2014, ApJ, 790, 41

Muraki, Y., Han, C., Bennett, D. P., et al. 2011, ApJ, 741, 22

Nataf, D. M., Gould, A., Fouqué, P., et al. 2013, ApJ, 769, 88

Orosz, J. A., Welsh, W. F., Carter, J. A., et al. 2012, Sci, 337, 1511

Poleski, R., Skowron, J., Udalski, A., et al. 2014, ApJ, 795, 42

Rhie, S. H. 1997, ApJ, 484, 63

Rhie, S. H. 2002, arXiv:astro-ph/0202294

Schechter, P. L., Mateo, M., \& Saha, A. 1993, PASP, 105, 1342

Sigurdsson, S., Richer, H. B., Hansen, B. M., Stairs, I. H., \& Thorsett, S. E. 2003, Sci, 301, 193

Silsbee, K., \& Rafikov, R. R. 2015, ApJ, 808, 58

Smullen, R. A., Kratter, K. M., \& Shannon, A. 2016, MNRAS, 461, 1288

Sumi, T., Kamiya, K., Bennett, D. P., et al. 2011, Natur, 473, 349

Sutherland, A. P., \& Fabrycky, D. C. 2016, ApJ, 818, 6

Szymański, M. K., Udalski, A., Soszyński, I., et al. 2011, AcA, 61, 83

Tomaney, A. B., \& Crotts, A. P. S. 1996, AJ, 112, 2872

Udalski, A. 2003, AcA, 53, 291

Udalski, A., Jung, Y. K., Han, C., et al. 2015, ApJ, 812, 47

Udalski, A., Szymański, M., Kałużny, J., et al. 1994, AcA, 44, 227

Verde, L., Peiris, H. V., Spergel, D. N., et al. 2003, ApJS, 148, 195

Welsh, W. F., Orosz, J. A., Carter, J. A., et al. 2012, Natur, 481, 475

Welsh, W. F., Orosz, J. A., Short, D. R., et al. 2015, ApJ, 809, 26

Wolszczan, A., \& Frail, D. A. 1992, Natur, 355, 145

Yee, J. C., Shvartzvald, Y., Gal-Yam, A., et al. 2012, ApJ, 755, 102 